\title{
Integrative Taxonomy, Phylogeny, and New Species of the Weevil Genus Onyxacalles Stüben (Coleoptera: Curculionidae: Cryptorhynchinae)
}

\author{
Peter E. Stüben ${ }^{1}$ and Jonas J. Astrin ${ }^{2}$ \\ ${ }^{1}$ Curculio Institute, Hauweg 62, 41066 Mönchengladbach, Germany \\ ${ }^{2}$ Zoologisches Forschungsmuseum Alexander Koenig (ZFMK), Molecular Taxonomy \& Biobank, Adenauerallee 160, \\ 53113 Bonn, Germany
}

Correspondence should be addressed to Peter E. Stüben, p.stueben@t-online.de

Received 21 September 2011; Revised 2 January 2012; Accepted 2 January 2012

Academic Editor: Brian Forschler

Copyright ( $) 2012$ P. E. Stüben and J. J. Astrin. This is an open access article distributed under the Creative Commons Attribution License, which permits unrestricted use, distribution, and reproduction in any medium, provided the original work is properly cited.

\begin{abstract}
A molecular phylogeny of the western Palearctic weevil genus Onyxacalles Stüben, 1999 is presented, combining two mitochondrial genes (COI and 16S) in a Bayesian analysis. Based on molecular data, Onyxacalles pyrenaeus Boheman, 1844 is transferred into the genus Kyklioacalles Stüben 1999 (K. fausti group) and-in an integrative taxonomy framework-the interaction between morphology and molecular analysis is illustrated. The species of Onyxacalles s. str. are assigned to three new species groups, $O$. henoni, O. luigionii, and O. portusveneris groups. The distribution of the related species in the Mediterranean area is illustrated with values of COI and $16 \mathrm{~S}$ p-distances. Three new species are described and distinguished from their related species: Onyxacalles nuraghi Stüben sp.n. from Italy (Sardinia), Onyxacalles torre Stüben and Astrin sp. n. from France (Corsica) and Onyxacalles vilae Stüben sp. n. from Croatia (Velebit Mts.). A catalogue of all 20 species of Onyxacalles is given, and a key is finally presented combined with image stacking of the habitus and aedeagus for all species.
\end{abstract}

\section{Introduction}

Together with a number of other genera, the genus Onyxacalles Stüben, 1999 (Curculionidae: Cryptorhynchinae) was separated by Stüben [1] from the formerly excessively broadly circumscribed genus Acalles Schoenherr, 1825 as a group with initially 8 species. Since then, many new species of Onyxacalles have been described, mainly from Spain and North Africa. These discoveries were supported by the morphological finding that the three species from the Canary Islands belong to this genus $[1,2]$, a thesis that gained support from recent molecular analysis and has contributed to the new subgenus Araneacalles Stüben and Astrin [3]. This closed a "biogeographical gap" (between the Pyrenees and northwestern Africa) as a direct consequence of target-oriented collecting activities and descriptions of many new Onyxacalles species over the past decade [4-9]. Thus, including the new species presented in this work, the genus now comprises 20 valid species.

Most species of Onyxacalles are found in the west Mediterranean area and on the Macaronesian Islands. Only one species, Onyxacalles croaticus (H. Brisout, 1897) [10], reaches Eastern Europe (Carpathians); another species, $O$. amasyaensis Wolf, 2001, was described from Turkey, but could be a synonym of Onyxacalles denominandus A. and F. Solari, 1907. This species richness in the west Mediterranean is well founded in the ecological preferences of Onyxcalles.

As "nocturnal goblins of the last primeval forests" [7], the species of Onyxacalles are not common in the often disturbed landscapes of the Iberian Peninsula and North Africa [11, 12]. These conditions provide good maps of relictual vegetation and information about the habitats, allowing us to trace these nocturnal Cryptorhynchinae in the dark and humid relicts of natural forests under big 


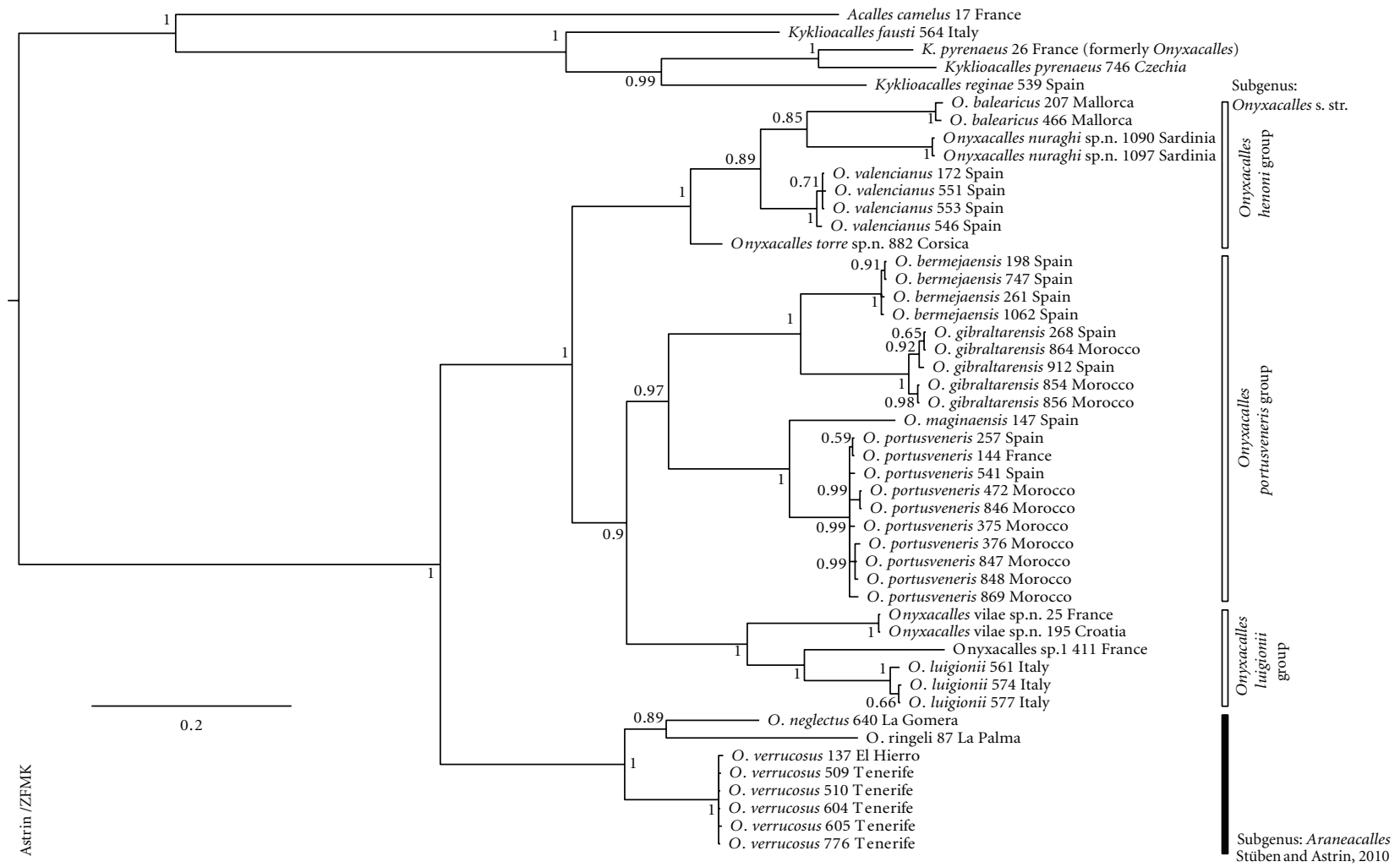

FIgURE 1: Bayesian consensus tree (50\% majority rule) for COI and $16 \mathrm{~S}$.

oak trees and behind a dense jungle of Smilax aspera L. Therefore, sifting by day is not the ideal method of catching Onyxacalles. With their long legs, Onyxacalles species climb trees by night and were fogged by us from the canopy of the laurisilva on the Canary Islands of Tenerife and La Gomera [13, 14]. The flightless Onyxacalles are highly specialised woodlander inhabitant, and because of their restricted dispersal (above all on the European continent), they are an ideal bioindicator of original forest, highlighting that protection and sustainable development should concern us as entomologists and conservationists.

Moreover, with the study of the cryptic and similar Onyxacalles species, the taxonomist, especially the morphologist, enters a minefield. Due to intraspecific variability, the few external characteristics that are suitable for a differential diagnosis do not guarantee a reliable (re)identification of species: if the tufts of bristles exist or not, if the elytra are short oval or elongated, or if the midgroove of pronotum is more or less distinct-all these are important pointers, but are not conclusive. All Onyxacalles species have the hooked apex of the aedeagus in common, as the genus name implies (cf. Figures 9(a)-9(1)). However, a complex internal sac of the aedeagus (endophallus) does not exist-as it is typical for Kyklioacalles, Dichromacalles, or most genera from Macaronesia. Here, we are quickly stretched to our limits of a descriptive morphology.

With the "Molecular Weevil Identification" Project (see below), we tread a path towards an integrative taxonomy [15-21]. Molecular taxonomy is not only an addition or an accessory "immunisation" to confirm morphological results. The integration of the two approaches must be done with the intention of falsifying and, if so, it can lead to a new way of viewing morphology: interspecific characteristics are discovered that are not regarded as belonging to intraspecific variability, or-more often-substituting the "human eyes," the way we look at things. Instead of "anthropocentric conspicuities" (e.g., forms, colours, sizes), we focus on constitutive characteristics (e.g., apomorphies and homologies) that were previously overlooked in our diagnostic keys. An example is the latest history of the science behind the original species Acalles pyrenaeus Boheman, 1844 (see below).

Another telling example is the present classification of Onyxacalles s. str. into two species groups: the O. luigioniiand $O$. pyrenaeus-groups, based on the bristles of the elytral intervals (cf. $[1,8])$. This hypothesis is no longer tenable, and this has nothing to do with superficial diagnosis of affinities or inaccurate observations. First, molecular analysis of related species reveals new informal species groups (see Figure 1) and makes evidence available to the morphologist, who then looks for new external characteristics. These are inconspicuous paradigm changes with a high impact [22], because the "puzzles," in this case the species, which were previously pressurised within the framework of "normal science" (and more and more frequently caused difficulties) continue, but the emphasis of the characteristics under the new molecular phylogenetic paradigma has changed (see "Catalogue of Onyxacalles"), and new characteristics are discovered (see "Key to the species of Onyxacalles"). 
The assumption that the morphologist should have seen a characteristic must be abandoned in favour of the question: would the morphologist be able to "see" it?

\section{Catalogue of Onyxacalles}

Species included in the molecular analysis are printed in bold (l.t. = type locality).

Genus: Onyxacalles Stüben, 1999a.

177 type species Acalles luigionii A. and F. Solari, 1907.

Subgenus: Onyxacalles s. str.

Onyxacalles henoni Group

balearicus Stüben, 2005: 115, Spain: Majorca (l.t.)

croaticus H. Brisout de Barneville, 1867: 62 (Acalles), Croatia (l.t.), Austria, Czech Republic, Germany, Poland, Slovakia, Slovenia

hannibali Germann, 2004: 118, Tunisia (l.t.)

henoni Bedel, 1888: 36 (Acalles), Algeria: Mt. Edough (1.t.).

nuraghi Stüben sp. n., Italy: Sardinia (1.t.).

torre Stüben and Astrin sp. n., France: Corsica (l.t.).

valencianus Germann, 2005: 104, Western Spain (l.t.).

Onyxacalles portusveneris Group

bermejaensis Stüben, 2001: 145, Spain (l.t.).

gibraltarensis Stüben, 2002: 206, Morocco (l.t.), Spain.

maginaensis Stüben, 2004: 120, Southern Spain (1.t.).

portusveneris Mayet, 1903: 74 (Acalles), France (1.t.), Spain, Morocco

seguraensis Stüben, 2003a: 201, Spain.

Onyxacalles luigionii Group

luigionii A. and F. Solari, 1907: 521 (Acalles), Central (l.t.) and Southern Italy.

vilae Stüben sp. n., Croatia (l.t.), France: Isère (perhaps Austria and Slovenia).

cf. luigionii, France: Alpes Maritimes.

Incertae Sedis

denominandus A. and F. Solari, 1907: 523 (Acalles), Turkey (l.t.).

porcheti Hoffmann, 1935: 162 (Acalles), France: Pyrenees (probably a synonym of $A$. luigionii, see also Stüben 2007: 149).

amasyaensis Wolf, 2001: 150, Turkey (1.t.). (probably a synonym of Acalles denominandus)

Subgenus: Araneacalles Stüben and Astrin, 2010: 78 type species Acalles verrucosus Wollaston, 1863.

neglectus Kulbe, 1999: 193, Canary Islands: La Gomera (l.t.), El Hierro. ringeli Kulbe 1999: 196, Canary Islands: La Palma (l.t.).

verrucosus Wollaston, 1863: 219 (Acalles), Canary Islands: Tenerife (l.t.), El Hierro.

\section{Materials and Methods}

The molecular analysis is based on 45 (43 after transfer of $O$. pyrenaeus, see below) individuals in 15 species or putative species of Onyxacalles and on 5 outgroup species (Cryptorhynchinae from 4 other genera; only the two closer genera are shown in the tree for better visualisation, while the 2 more distant ones were removed; see Table 1). Most sequences have been published in Astrin et al. (2012). Collecting and vouchering information as well as GenBank accession numbers are given in Table 1. Voucher specimens and extracted genomic DNA are deposited at the Biobank of the ZFMK (Zoologisches Forschungsmuseum Alexander Koenig, Bonn, Germany).

DNA extraction was carried out on samples preserved in ethanol or on dried material, using Macherey-Nagel Nucleo Spin Tissue kits (Dueren, Germany) or BioSprint 96 kits (Qiagen, Hilden, Germany). We extracted DNA from either 2-3 legs, head and prothorax, or sometimes also the whole weevil, depending on size and conservation of the sample. PCR reaction mixes $(50 \mu \mathrm{L})$ contained $125 \mathrm{nmol} \mathrm{MgCl}_{2}, 5 \mu \mathrm{L}$ 10x PCR-buffer, $25 \mathrm{pmol}$ of forward and reverse primer each, 5 pmol dNTPs, 1.75 units of Taq polymerase, and $5 \mu \mathrm{L}$ total undiluted DNA template. The lab chemicals were purchased from Sigma-Aldrich (Steinheim, Germany). We used the Qiagen (Hilden, Germany) Multiplex PCR kit in cases where the regular protocol failed. PCR primers were taken from Astrin and Stüben (2008; COI is based on the Folmer et al. [23] region; 16S is based on the Crandall and Fitzpatrick (1996) region). Primer sequences were as follows: LCOI490JJ (COI forward, fw) 5' -CHACWAAYCATAAAGATATYGG-3', HCO2198-JJ (COI reverse, rev) 5'-AWACTTCVGGRTGVCCAAARAATCA-3'; 16S-ar-JJ (16S fw, erroneously as "rev" in [24]) 5'-CRCCTGTTTATTAAAAACAT-3', 16S1472-JJ (16S rev) 5' -AGATAGAAACCRACCTGG-3'. Thermal cycling was performed on blocks of the type GeneAmp PCR System 2700 (Applied Biosystems, Foster City, CA, USA). PCR program for $16 \mathrm{~S}$ : first cycle set (15 repeats): $35 \mathrm{~s}$ denaturation at $94^{\circ} \mathrm{C}, 35 \mathrm{~s}$ annealing at $55^{\circ} \mathrm{C}\left(-1^{\circ} \mathrm{C}\right.$ per cycle) and $60 \mathrm{~s}$ extension at $72^{\circ} \mathrm{C}$. Second cycle set $(25$ repeats): $35 \mathrm{~s}$ denaturation at $94^{\circ} \mathrm{C}, 35 \mathrm{~s}$ annealing at $40^{\circ} \mathrm{C}$, and $50 \mathrm{~s}$ extension at $72^{\circ} \mathrm{C}$. PCR program for COI: same as for $16 \mathrm{~S}$, but annealing temperatures at $70^{\circ} \mathrm{C}$ and $55^{\circ} \mathrm{C}$, with a decrease of $2^{\circ} \mathrm{C}$ per cycle in the first cycle set. Doublestranded sequencing was carried out by a sequencing facility (Macrogen, South Korea, and Netherlands) using the same primers as in PCR.

DNA sequence alignment was performed manually (COI) or using the MUSCLE ver. 3.6 programme [28] (16S), run with default parameters. Sequence length was 554 bp for $16 \mathrm{~S}$ (aligned; longest sequence: $544 \mathrm{bp}$; shortest: $533 \mathrm{bp}$ ) and $658 \mathrm{bp}$ for COI, for concatenated sequence data $1212 \mathrm{bp}$. The $16 \mathrm{~S}$ alignment comprised 29 positions with 
TABLE 1: Collecting data, vouchers, and GenBank accession numbers for the material analysed in this study. All specimens determined by P. E. Stüben, 2010 and 2011. Vouchers (DNA, morphology) are kept at the ZFMK Biobank. Most sequences have been published (or are reviewed) in Astrin et al. 2012 [21]. GenBank accession numbers of new sequences in this study start with "JN..." Taxonomic changes with regard to this publication are printed in brackets (old name).

\begin{tabular}{|c|c|c|c|}
\hline Taxon & Collecting data & DNA voucher & COI $16 S$ \\
\hline $\begin{array}{l}\text { Acalles camelus } \\
\text { (Fabricius } 1792 \text { ) }\end{array}$ & $\begin{array}{l}\text { France: Isère, } 2 \mathrm{~km} \text { SE Lans en Vercors, Montagne de } \\
\text { Lans; N } 45^{\circ} 06^{\prime} 45^{\prime \prime} \text { E } 05^{\circ} 36^{\prime} 21^{\prime \prime}, 1352 \mathrm{~m} \text {; Abies, Fagus, } \\
\text { Fraxinus, 2005, Stüben }\end{array}$ & $\begin{array}{l}\text { ZFMK-DNA-JJ0017, } \\
\text { ZFMK-TIS-cI0026cam }\end{array}$ & $\begin{array}{l}\text { EU286282 } \\
\text { EU286447 }\end{array}$ \\
\hline $\begin{array}{l}\text { Acallorneuma doderoi A. } \\
\text { and F. Solari } 1908\end{array}$ & 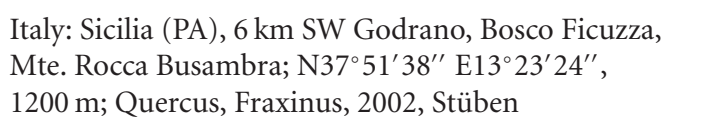 & $\begin{array}{l}\text { ZFMK-DNA-JJ0065, } \\
\text { ZFMK-TIS-cS0082dod }\end{array}$ & $\begin{array}{l}\text { EU286292 } \\
\text { EU286457 }\end{array}$ \\
\hline $\begin{array}{l}\text { Cryptorhynchus lapathi } \\
\text { (Linné 1758) }\end{array}$ & Germany: Bienen bei Rees, Altrheinarm, 2004, Scharf & $\begin{array}{l}\text { ZFMK-DNA-JJ0214, } \\
\text { ZFMK-TIS-cD0354lap }\end{array}$ & $\begin{array}{l}\text { EU286360 } \\
\text { EU286523 }\end{array}$ \\
\hline $\begin{array}{l}\text { Kyklioacalles fausti } \\
\text { (Meyer 1896) [25] }\end{array}$ & $\begin{array}{l}\text { Italy: Campania, Cilento, } 6 \mathrm{~km} \text { SE Vallo d. Lucania, M. } \\
\text { Sacro o Gelbison; N } 40^{\circ} 12^{\prime} 41^{\prime \prime} \text { E } 15^{\circ} 19^{\prime} 42^{\prime \prime}, 1544 \mathrm{~m} \text {; } \\
\text { Fagus, 2008, Stüben }\end{array}$ & $\begin{array}{l}\text { ZFMK-DNA-JJ0564, } \\
\text { ZFMK-TIS-cI625fau }\end{array}$ & $\begin{array}{l}\text { GU213776 } \\
\text { GU213772 }\end{array}$ \\
\hline $\begin{array}{l}\text { Kyklioacalles pyrenaeus } \\
\text { (Boheman 1844) [26] } \\
\text { (gen. Onyxacalles) }\end{array}$ & $\begin{array}{l}\text { France: Isère, } 14 \mathrm{~km} \mathrm{~N} \text { Grenoble, Massif de la } \\
\text { Chartreuse, NW Col de Porte; N } 45^{\circ} 18^{\prime} 40^{\prime \prime} \text { E } 05^{\circ} 45^{\prime} 17^{\prime \prime} \text {, } \\
1649 \text { m; Abies, Fagus, Fraxinus, 2005, Stüben }\end{array}$ & $\begin{array}{l}\text { ZFMK-DNA-JJ0026, } \\
\text { ZFMK-TIS-cI0035pyr }\end{array}$ & $\begin{array}{l}\text { GU988172 } \\
\text { GU987762 }\end{array}$ \\
\hline $\begin{array}{l}\text { Kyklioacalles pyrenaeus [26] } \\
\text { (gen. Onyxacalles) }\end{array}$ & $\begin{array}{l}\text { Czech Republic: W Bohemia (KT), Balkovy, Doubrava } \\
\text { Hill (6545), 2008, Kresl }\end{array}$ & $\begin{array}{l}\text { ZFMK-DNA-JJ0764, } \\
\text { ZFMK-TIS-cCz798pyr }\end{array}$ & $\begin{array}{l}\text { GU981555 } \\
\text { GU981506 }\end{array}$ \\
\hline $\begin{array}{l}\text { Kyklioacalles reginae Stüben } \\
2003\end{array}$ & $\begin{array}{l}\text { Spain: Teruel, S. Javalambre, Fuente la Risca near Arcos } \\
\text { de las Salinas; N } 39^{\circ} 59^{\prime} 56^{\prime \prime} \text { W } 01^{\circ} 01^{\prime} 21^{\prime \prime}, 1121 \mathrm{~m} \text {; } \\
\text { Amelanchier ovalis, Acer monspessulanum, Erinacea } \\
\text { anthyllis, Ulex, 2008, Stüben }\end{array}$ & $\begin{array}{l}\text { ZFMK-DNA-JJ0539, } \\
\text { ZFMK-TIS-cE600reg }\end{array}$ & $\begin{array}{l}\text { GU981544 } \\
\text { GU981495 }\end{array}$ \\
\hline $\begin{array}{l}\text { Onyxacalles balearicus } \\
\text { Stüben } 2005\end{array}$ & $\begin{array}{l}\text { Spain: Mallorca, } 3 \mathrm{~km} \text { SE Lluc, Sra. de Tramuntana, Sa } \\
\text { Maleta; N39 } 48^{\prime} 47^{\prime \prime} \text { E } 02^{\circ} 53^{\prime} 23^{\prime \prime}, 571 \mathrm{~m} \text {; Quercus ilex, } \\
\text { 2004, Stüben }\end{array}$ & $\begin{array}{l}\text { ZFMK-DNA-JJ0207, } \\
\text { ZFMK-TIS-cE0168bal }\end{array}$ & $\begin{array}{l}\text { EU286357 } \\
\text { EU286521 }\end{array}$ \\
\hline $\begin{array}{l}\text { Onyxacalles balearicus } \\
\text { Stüben } 2005\end{array}$ & $\begin{array}{l}\text { Spain: Mallorca, } 11 \mathrm{~km} \text { NE Lluc, Sra. de Tramuntana; } \\
\text { N39 } 52^{\prime} 03^{\prime \prime} \text { E } 02^{\circ} 58^{\prime} 20^{\prime \prime}, 107 \mathrm{~m} \text {; PT, Smilax aspera, } \\
\text { Quercus ilex, 2004, Stüben }\end{array}$ & $\begin{array}{l}\text { ZFMK-DNA-JJ0466, } \\
\text { ZFMK-TIS-cE0294bal }\end{array}$ & GU988348 \\
\hline $\begin{array}{l}\text { Onyxacalles bermejaensis } \\
\text { Stüben } 2001\end{array}$ & $\begin{array}{l}\text { Spain: Andalucía, } 11 \mathrm{~km} \text { S Ronda, Sierra de las Nieves; } \\
\text { N36 } 39^{\prime} 51^{\prime \prime} \text { W } 05^{\circ} 05^{\prime} 01^{\prime \prime}, 1047 \mathrm{~m} \text {; Quercus ilex, 2005, } \\
\text { Stüben }\end{array}$ & $\begin{array}{l}\text { ZFMK-DNA-JJ0198, } \\
\text { ZFMK-TIS-cE0167ber }\end{array}$ & $\begin{array}{l}\text { EU286350 } \\
\text { EU286514 }\end{array}$ \\
\hline $\begin{array}{l}\text { Onyxacalles bermejaensis } \\
\text { Stüben } 2001\end{array}$ & $\begin{array}{l}\text { Spain: Málaga, } 9 \mathrm{~km} \text { SE Ubrique, Sierra de Líbar; } \\
\text { N36 } 36^{\prime} 52^{\prime \prime} \text { W } 05^{\circ} 23^{\prime} 16^{\prime \prime}, 663 \text { m; Quercus ilex, } \\
\text { Ceratonia, 2007, Stüben }\end{array}$ & $\begin{array}{l}\text { ZFMK-DNA-JJ0261, } \\
\text { ZFMK-TIS-cE0194ber }\end{array}$ & $\begin{array}{l}\text { GU988244 } \\
\text { GU987827 }\end{array}$ \\
\hline $\begin{array}{l}\text { Onyxacalles bermejaensis } \\
\text { Stüben } 2001\end{array}$ & $\begin{array}{l}\text { Spain: Málaga, NW Marbella, Sierra de las Nieves; } \\
\text { N36 } 39^{\prime} 52^{\prime \prime} \text { W } 05^{\circ} 04^{\prime} 57^{\prime \prime}, 1043 \text { m; Quercus ilex, } \\
\text { Echinodera spinosa, 2009, Stüben }\end{array}$ & $\begin{array}{l}\text { ZFMK-DNA-JJ0747, } \\
\text { ZFMK-TIS-cE778ber }\end{array}$ & $\begin{array}{l}\text { GU988506 } \\
\text { GU988066 }\end{array}$ \\
\hline $\begin{array}{l}\text { Onyxacalles bermejaensis } \\
\text { Stüben } 2001\end{array}$ & $\begin{array}{l}\text { Spain: Prov. Málaga, Algatocín, near Opayar; } \\
\text { N36 } 34^{\prime} 39^{\prime \prime} \text { W } 05^{\circ} 18^{\prime} 13^{\prime \prime}, 576 \text { m, Quercus sp., } \\
\text { 17.8.2010, Stüben }\end{array}$ & $\begin{array}{l}\text { ZFMK-DNA-JJ1062, } \\
\text { ZFMK-TIS-cES1062 }\end{array}$ & $\begin{array}{l}\text { JN121398 } \\
--------\end{array}$ \\
\hline $\begin{array}{l}\text { Onyxacalles gibraltarensis } \\
\text { Stüben } 2002\end{array}$ & $\begin{array}{l}\text { Spain: Cádiz, } 10 \text { km SW Algeciras, El Bujeo; } \\
\text { N36 } 04^{\prime} 10^{\prime \prime} \text { W05³ } 31^{\prime} 48^{\prime \prime}, 257 \text { m; Quercus suber, 2007, } \\
\text { Stüben }\end{array}$ & $\begin{array}{l}\text { ZFMK-DNA-JJ0268, } \\
\text { ZFMK-TIS-cE0206gib }\end{array}$ & $\begin{array}{l}\text { GU988249 } \\
\text { GU987832 }\end{array}$ \\
\hline $\begin{array}{l}\text { Onyxacalles gibraltarensis } \\
\text { Stüben } 2002\end{array}$ & $\begin{array}{l}\text { Morocco: Rif, SW Oued-Laou, river, O. Laou; } \\
\text { N351' } 47^{\prime \prime} \text { W05 } 13^{\prime} 38^{\prime \prime}, 210 \text { m; Quercus suber, } \\
\text { Smilax, Arbutus, } 2009 \text {, Stüben }\end{array}$ & $\begin{array}{l}\text { ZFMK-DNA-JJ0854, } \\
\text { ZFMK-TIS-cE897gib }\end{array}$ & $\begin{array}{l}\text { GU988577 } \\
\text { GU988137 }\end{array}$ \\
\hline $\begin{array}{l}\text { Onyxacalles gibraltarensis } \\
\text { Stüben } 2002\end{array}$ & $\begin{array}{l}\text { Morocco: W Sebta, vir. Biutz; N355 } 53^{\prime} 04^{\prime \prime} \text { W0 } 05^{\circ} 24^{\prime} 08^{\prime \prime} \text {, } \\
337 \text { m; Quercus suber, Smilax, Arbutus, } 2009 \text {, Stüben }\end{array}$ & $\begin{array}{l}\text { ZFMK-DNA-JJ0856, } \\
\text { ZFMK-TIS-cE899gib }\end{array}$ & $\begin{array}{l}\text { GU988578 } \\
\text { GU988138 }\end{array}$ \\
\hline $\begin{array}{l}\text { Onyxacalles gibraltarensis } \\
\text { Stüben } 2002\end{array}$ & $\begin{array}{l}\text { Morocco: S Ksar-es-Seghir; N354ㄴㄷㄴ } 16^{\prime \prime} \text { W } 05^{\circ} 30^{\prime} 49^{\prime \prime} \text {, } \\
278 \text { m; Pistacia, Quercus suber, 2009, Stüben }\end{array}$ & $\begin{array}{l}\text { ZFMK-DNA-JJ0864, } \\
\text { ZFMK-TIS-cE907gib }\end{array}$ & $\begin{array}{l}\text { GU988584 } \\
\text { GU988144 }\end{array}$ \\
\hline $\begin{array}{l}\text { Onyxacalles gibraltarensis } \\
\text { Stüben } 2002\end{array}$ & $\begin{array}{l}\text { Spain: Cádiz, Los Barrios, Alcornocales N.P., between } \\
\text { Facinos, Río Las Cañas and Mantera Torero; Olea } \\
\text { europaea, 2009, Torres }\end{array}$ & $\begin{array}{l}\text { ZFMK-DNA-JJ0912, } \\
\text { ZFMK-TIS-cE949gib }\end{array}$ & GU988608 \\
\hline $\begin{array}{l}\text { Onyxacalles luigionii } \\
\text { (A. \& F. Solari 1907) }\end{array}$ & $\begin{array}{l}\text { Italy: Campania, Cilento, } 6 \mathrm{~km} \text { SE Vallo d. Lucania, M. } \\
\text { Sacro o Gelbison; N } 40^{\circ} 12^{\prime} 41^{\prime \prime} \text { E } 15^{\circ} 19^{\prime} 42^{\prime \prime}, 1544 \mathrm{~m} \text {; } \\
\text { Fagus, 2008, Stüben }\end{array}$ & $\begin{array}{l}\text { ZFMK-DNA-JJ0561, } \\
\text { ZFMK-TIS-cI622lui }\end{array}$ & $\begin{array}{l}\text { GU988407 } \\
\text { GU987967 }\end{array}$ \\
\hline
\end{tabular}


TABle 1: Continued.

\begin{tabular}{|c|c|c|c|}
\hline Taxon & Collecting data & DNA voucher & COI $16 \mathrm{~S}$ \\
\hline $\begin{array}{l}\text { Onyxacalles luigionii } \\
\text { (A. \& F. Solari 1907) }\end{array}$ & $\begin{array}{l}\text { Italy: Campania, Monti Picentini, } 9 \mathrm{~km} \text { N Acerno, } \\
\text { Piano Laceno; } 440^{\circ} 48^{\prime} 58^{\prime \prime} \mathrm{E} 15^{\circ} 07^{\prime} 35^{\prime \prime}, 1210 \mathrm{~m} \text {; Fagus, } \\
\text { 2008, Stüben }\end{array}$ & $\begin{array}{l}\text { ZFMK-DNA-JJ0574, } \\
\text { ZFMK-TIS-cI635lui }\end{array}$ & $\begin{array}{l}\text { GU988417 } \\
\text { GU987977 }\end{array}$ \\
\hline $\begin{array}{l}\text { Onyxacalles luigionii } \\
\text { (A. \& F. Solari 1907) }\end{array}$ & $\begin{array}{l}\text { Italy: Basilicata, Monte Pollino, } 9 \text { km SE Rotonda, Rif. } \\
\text { de Gasperi; N39 } 54^{\prime} 37^{\prime \prime} \text { E16 } 07^{\prime} 15^{\prime \prime}, 1486 \text { m; Fagus, } \\
\text { 2008, Stüben }\end{array}$ & $\begin{array}{l}\text { ZFMK-DNA-JJ0577, } \\
\text { ZFMK-TIS-cI638lui }\end{array}$ & $\begin{array}{l}\text { GU988418 } \\
\text { GU987979 }\end{array}$ \\
\hline $\begin{array}{l}\text { Onyxacalles maginaensis } \\
\text { Stüben } 2004\end{array}$ & $\begin{array}{l}\text { Spain: Andalucía, } 28 \text { km E Jaén, Sierra Magina; } \\
\text { N37 } 43^{\prime} 21^{\prime \prime} \text { W03 } 29^{\prime} 11^{\prime \prime}, 1600 \text { m; Quercus ilex, 2005, } \\
\text { Stüben }\end{array}$ & $\begin{array}{l}\text { ZFMK-DNA-JJ0147, } \\
\text { ZFMK-TIS-cE0169mag }\end{array}$ & $\begin{array}{l}\text { EU286327 } \\
\text { EU286491 }\end{array}$ \\
\hline $\begin{array}{l}\text { Onyxacalles maginaensis } \\
\text { Stüben } 2004\end{array}$ & $\begin{array}{l}\text { Spain: Almería, } 11 \mathrm{~km} \text { NW Laujar de Andarax, Sierra } \\
\text { Nevada, Bayárcal; N37 } 02^{\prime} 27^{\prime \prime} \text { W03 } 00^{\prime} 12^{\prime \prime}, 1291 \mathrm{~m} ; \\
\text { Quercus ilex, 2007, Stüben }\end{array}$ & $\begin{array}{l}\text { ZFMK-DNA-JJ0257, } \\
\text { ZFMK-TIS-cE0187mag }\end{array}$ & $\begin{array}{l}\text { submitted to } \\
\text { GenBank }\end{array}$ \\
\hline $\begin{array}{l}\text { Onyxacalles maginaensis } \\
\text { Stüben } 2004\end{array}$ & $\begin{array}{l}\text { Spain: Teruel, S. Javalambre, Fuente la Risca near Arcos } \\
\text { de las Salinas; N } 39^{\circ} 59^{\prime} 56^{\prime \prime} \text { W } 01^{\circ} 01^{\prime} 21^{\prime \prime}, 1121 \mathrm{~m} \text {; } \\
\text { Amelanchier ovalis, Acer monspessulanum, Erinacea } \\
\text { anthyllis, Ulex, 2008, Stüben }\end{array}$ & $\begin{array}{l}\text { ZFMK-DNA-JJ0541, } \\
\text { ZFMK-TIS-cE602mag }\end{array}$ & $\begin{array}{l}\text { GU988390 } \\
\text { GU987950 }\end{array}$ \\
\hline $\begin{array}{l}\text { Onyxacalles neglectus Kulbe } \\
1999\end{array}$ & $\begin{array}{l}\text { Spain: Canary Islands, La Gomera, S Hermigua, El } \\
\text { Cedro, Las Mimbreras; N2 } 28^{\circ} 07^{\prime} 27^{\prime \prime} \mathrm{W} 17^{\circ} 13^{\prime} 26^{\prime \prime} \text {, } \\
901 \mathrm{~m} \text {; laurisilva, 2008, Astrin and Stüben }\end{array}$ & $\begin{array}{l}\text { ZFMK-DNA-JJ0640, } \\
\text { ZFMK-TIS-cE713neg }\end{array}$ & $\begin{array}{l}\text { FJ716525 } \\
\text { GU988014 }\end{array}$ \\
\hline Onyxacalles nuraghi sp.n. & $\begin{array}{l}\text { Italy: W-Sardinia, E Macomer: above Lei; N } 40^{\circ} 19^{\prime} 54^{\prime \prime} \\
\text { E08 } 53^{\prime} 49^{\prime \prime}, 1020 \text { m; Quercus, Acer monspessulanum, } \\
\text { 4.10.2010, Stüben }\end{array}$ & $\begin{array}{l}\text { ZFMK-DNA-JJ1090, } \\
\text { ZFMK-TIS-cIT1090 }\end{array}$ & $\begin{array}{l}\text { JN642097 } \\
\text { JN121399 }\end{array}$ \\
\hline Onyxacalles nuraghi sp.n. & $\begin{array}{l}\text { Italy: W-Sardinia, E Macomer: above Lei; N } 40^{\circ} 19^{\prime} 17^{\prime \prime} \\
\text { E08 } 53^{\prime} 52^{\prime \prime}, 586 \mathrm{~m} \text {; Quercus ilex, 7.10.2010, Stüben }\end{array}$ & $\begin{array}{l}\text { ZFMK-DNA-JJ1097, } \\
\text { ZFMK-TIS-cIT1097 }\end{array}$ & $\begin{array}{l}\text { JN642098 } \\
\text { JN121300 }\end{array}$ \\
\hline $\begin{array}{l}\text { Onyxacalles portusveneris } \\
\text { (Mayet 1903) [27] }\end{array}$ & $\begin{array}{l}\text { France: Gard, } 15 \mathrm{~km} \text { NE Nimes, Pont du Gard, Collias; } \\
\text { N } 43^{\circ} 57^{\prime} 03^{\prime \prime} \text { E } 04^{\circ} 28^{\prime} 59^{\prime \prime}, 68 \text { m; Quercus ilex, 2006, } \\
\text { Stüben }\end{array}$ & $\begin{array}{l}\text { ZFMK-DNA-JJ0144, } \\
\text { ZFMK-TIS-cF0166por }\end{array}$ & $\begin{array}{l}\text { EU286326 } \\
\text { EU286490 }\end{array}$ \\
\hline $\begin{array}{l}\text { Onyxacalles portusveneris } \\
\text { (Mayet 1903) [27] }\end{array}$ & 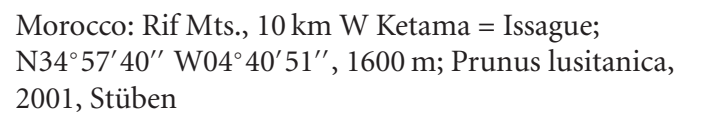 & $\begin{array}{l}\text { ZFMK-DNA-JJ0375, } \\
\text { ZFMK-TIS-cM480por }\end{array}$ & GU988311 \\
\hline $\begin{array}{l}\text { Onyxacalles portusveneris } \\
\text { (Mayet 1903) [27] }\end{array}$ & 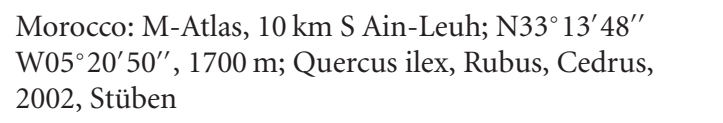 & $\begin{array}{l}\text { ZFMK-DNA-JJ0376, } \\
\text { ZFMK-TIS-cM481por }\end{array}$ & GU988312 \\
\hline $\begin{array}{l}\text { Onyxacalles portusveneris } \\
\text { (Mayet 1903) [27] }\end{array}$ & $\begin{array}{l}\text { Morocco: High Atlas, E Marrakech, N Taddert, (near } \\
\text { Tazouguerte); N } 31^{\circ} 28^{\prime} 07^{\prime \prime} \text { W } 07^{\circ} 24^{\prime} 59^{\prime \prime}, 1727 \mathrm{~m} \text {; } \\
\text { Quercus, 2009, Stüben }\end{array}$ & $\begin{array}{l}\text { ZFMK-DNA-JJ0846, } \\
\text { ZFMK-TIS-cE889port }\end{array}$ & $\begin{array}{l}\text { GU988573 } \\
\text { GU988133 }\end{array}$ \\
\hline $\begin{array}{l}\text { Onyxacalles portusveneris } \\
\text { (Mayet 1903) [27] }\end{array}$ & 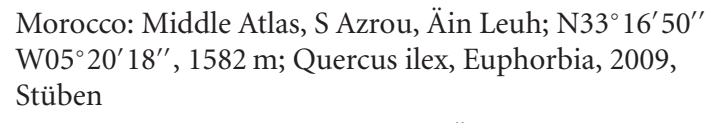 & $\begin{array}{l}\text { ZFMK-DNA-JJ0847, } \\
\text { ZFMK-TIS-cE890port }\end{array}$ & $\begin{array}{l}\text { GU988574 } \\
\text { GU988134 }\end{array}$ \\
\hline $\begin{array}{l}\text { Onyxacalles portusveneris } \\
\text { (Mayet 1903) [27] }\end{array}$ & $\begin{array}{l}\text { Morocco: Middle Atlas, S Azrou, S Äin Leuh; } \\
\text { N33 } 14^{\prime} 57^{\prime \prime} \text { W05 } 21^{\prime} 04^{\prime \prime}, 1715 \text { m; Quercus ilex, 2009, } \\
\text { Stüben }\end{array}$ & $\begin{array}{l}\text { ZFMK-DNA-JJ0848, } \\
\text { ZFMK-TIS-cE891port }\end{array}$ & $\begin{array}{l}\text { GU988575 } \\
\text { GU988135 }\end{array}$ \\
\hline $\begin{array}{l}\text { Onyxacalles portusveneris } \\
\text { (Mayet 1903) [27] }\end{array}$ & $\begin{array}{l}\text { Morocco: Rif, } 10 \mathrm{~km} \text { W Ketama; N34 } 57^{\prime} 40^{\prime \prime} \\
\text { W04 } 40^{\prime} 51^{\prime \prime}, 1600 \mathrm{~m} \text {; Cedrus, Prunus, 2009, Stüben }\end{array}$ & $\begin{array}{l}\text { ZFMK-DNA-JJ0869, } \\
\text { ZFMK-TIS-cE912port }\end{array}$ & $\begin{array}{l}\text { GU988587 } \\
\text { GU988147 }\end{array}$ \\
\hline $\begin{array}{l}\text { Onyxacalles portusveneris } \\
\text { (Mayet 1903) [27] } \\
\text { (Onyxacalles } \mathrm{sp} . \text { ) }\end{array}$ & $\begin{array}{l}\text { Morocco: High Atlas, } 59 \mathrm{~km} \text { SE Marrakech; } \\
\mathrm{N} 31^{\circ} 28^{\prime} 19^{\prime \prime} \mathrm{W} 07^{\circ} 24^{\prime} 22^{\prime \prime}, 1500 \mathrm{~m} \text {; Quercus ilex, } \\
\text { Quercus suber, 2002, Stüben }\end{array}$ & $\begin{array}{l}\text { ZFMK-DNA-JJ0472, } \\
\text { ZFMK-TIS-cM482mag }\end{array}$ & $\begin{array}{l}\text { GU988350 } \\
\text { GU987922 }\end{array}$ \\
\hline $\begin{array}{l}\text { Onyxacalles ringeli Kulbe } \\
1999\end{array}$ & $\begin{array}{l}\text { Spain: Canary Islands, La Palma, Cumbre Nueva, } \\
4,5 \text { km SE El Paso, El Pilar; N28 } 37^{\prime} 37^{\prime \prime} \text { W } 17^{\circ} 49^{\prime} 45^{\prime \prime} \text {, } \\
1432 \text { m; laurisilva, 2006, Stüben }\end{array}$ & $\begin{array}{l}\text { ZFMK-DNA-JJ0087, } \\
\text { ZFMK-TIS-cC0171rin }\end{array}$ & $\begin{array}{l}\text { EU286300 } \\
\text { EU286465 }\end{array}$ \\
\hline $\begin{array}{l}\text { Onyxacalles sp. } 1 \\
\text { (O. luigionii) }\end{array}$ & $\begin{array}{l}\text { France: Alpes-Maritimes, } 3 \mathrm{~km} \text { W Sospel, Col de Braus; } \\
\text { N43 } 52^{\prime} 34^{\prime \prime} \text { E } 07^{\circ} 24^{\prime} 17^{\prime \prime}, 1051 \mathrm{~m} \text {; Quercus pubescens, } \\
\text { Ostrya carpinifolia, broom, } 2007 \text {, Stüben }\end{array}$ & $\begin{array}{l}\text { ZFMK-DNA-JJ0411, } \\
\text { ZFMK-TIS-cF440lui }\end{array}$ & $\begin{array}{l}\text { GU988325 } \\
\text { GU987897 }\end{array}$ \\
\hline $\begin{array}{l}\text { Onyxacalles torre sp. } \mathrm{n} . \\
(\text { O. henoni) }\end{array}$ & $\begin{array}{l}\text { France: Corsica, Col de Vizzavona, } 22 \text { km S Corte; } \\
\text { N42 } 06^{\prime} 45^{\prime \prime} \text { E09 } 06^{\prime} 49^{\prime \prime}, 1100 \text { m; Fagus, 2001, Stüben }\end{array}$ & $\begin{array}{l}\text { ZFMK-DNA-JJ0882, } \\
\text { ZFMK-TIS-cF479hen }\end{array}$ & GU988592 \\
\hline
\end{tabular}


TABLE 1: Continued.

\begin{tabular}{|c|c|c|c|}
\hline Taxon & Collecting data & DNA voucher & COI $16 \mathrm{~S}$ \\
\hline $\begin{array}{l}\text { Onyxacalles valencianus } \\
\text { Germann } 2005\end{array}$ & $\begin{array}{l}\text { Spain: Alicante, } 7 \text { km SW Alcoi, Sierra de Menechaor, } \\
\text { Santurio de la Font Roja; N } 38^{\circ} 39^{\prime} 34^{\prime \prime} \\
\text { W00 } 32^{\prime} 29^{\prime \prime}, 1296 \text { m; Quercus ilex, } 2007 \text {, Stüben }\end{array}$ & $\begin{array}{l}\text { ZFMK-DNA-JJ0172, } \\
\text { ZFMK-TIS-cE0180val }\end{array}$ & $\begin{array}{l}\text { EU286331 } \\
\text { EU286495 }\end{array}$ \\
\hline $\begin{array}{l}\text { Onyxacalles valencianus } \\
\text { Germann } 2005\end{array}$ & $\begin{array}{l}\text { Spain: Castellón, Morella, Barranco de la Bota; } \\
\text { N } 40^{\circ} 33^{\prime} 12^{\prime \prime} \text { W } 00^{\circ} 00^{\prime} 27^{\prime \prime}, 814 \text { m; Quercus ilex, Hedera } \\
\text { helix, 2008, Stüben }\end{array}$ & $\begin{array}{l}\text { ZFMK-DNA-JJ0546, } \\
\text { ZFMK-TIS-cE607val }\end{array}$ & $\begin{array}{l}\text { GU988393 } \\
\text { GU987953 }\end{array}$ \\
\hline $\begin{array}{l}\text { Onyxacalles valencianus } \\
\text { Germann } 2005\end{array}$ & $\begin{array}{l}\text { Spain: Barcelona, above dry river bed, near Vallirana; } \\
\mathrm{N} 41^{\circ} 22^{\prime} 36^{\prime \prime} \text { E } 01^{\circ} 55^{\prime} 02^{\prime \prime}, 245 \mathrm{~m} \text {; Quercus ilex, Ficus } \\
\text { carica, Smilax aspera, } 2008 \text {, Stüben }\end{array}$ & $\begin{array}{l}\text { ZFMK-DNA-JJ0551, } \\
\text { ZFMK-TIS-cE612val }\end{array}$ & $\begin{array}{l}\text { GU988398 } \\
\text { GU987958 }\end{array}$ \\
\hline $\begin{array}{l}\text { Onyxacalles valencianus } \\
\text { Germann } 2005\end{array}$ & $\begin{array}{l}\text { spain: Barcelona, S. Montseny, Tordera valley, near St. } \\
\text { Marçal; N } 41^{\circ} 48^{\prime} 01^{\prime \prime} \text { E02 } 22^{\circ} 15^{\prime \prime}, 1060 \text { m, 2008, Stüben }\end{array}$ & $\begin{array}{l}\text { ZFMK-DNA-JJ0553, } \\
\text { ZFMK-TIS-cE614val }\end{array}$ & $\begin{array}{l}\text { GU988400 } \\
\text { GU987960 }\end{array}$ \\
\hline $\begin{array}{l}\text { Onyxacalles verrucosus } \\
\text { (Wollaston 1863) }\end{array}$ & $\begin{array}{l}\text { Spain: Canary Islands, El Hierro, } 7 \text { km W La Frontera, } \\
\text { Pista Derrabado; N } 27^{\circ} 44^{\prime} 29^{\prime \prime} \text { W } 18^{\circ} 03^{\prime} 24^{\prime \prime}, 895 \mathrm{~m} \text {; } \\
\text { Laurus azorica, 2006, Stüben }\end{array}$ & $\begin{array}{l}\text { ZFMK-DNA-JJ0137, } \\
\text { ZFMK-TIS-cC0170ver }\end{array}$ & $\begin{array}{l}\text { EU286324 } \\
\text { EU286488 }\end{array}$ \\
\hline $\begin{array}{l}\text { Onyxacalles verrucosus } \\
\text { (Wollaston 1863) }\end{array}$ & $\begin{array}{l}\text { Spain: Canary Islands, Tenerife, } 6 \mathrm{~km} \text { N La Laguna, } \\
\text { Monte de las Mercedes; N28 } 31^{\prime} 50^{\prime \prime} \mathrm{W} 16^{\circ} 17^{\prime} 09^{\prime \prime} \text {, } \\
950 \mathrm{~m} \text {; laurisilva, 2003, Stüben }\end{array}$ & $\begin{array}{l}\text { ZFMK-DNA-JJ0509, } \\
\text { ZFMK-TIS-cE570ver }\end{array}$ & GU987937 \\
\hline $\begin{array}{l}\text { Onyxacalles verrucosus } \\
\text { (Wollaston } 1863 \text { ) }\end{array}$ & $\begin{array}{l}\text { Spain: Canary Islands, Tenerife, } 4 \text { km S Los Silos, Teno } \\
\text { Mts., Monte del Aqua; N2 } 28^{\circ} 19^{\prime} 20^{\prime \prime} \mathrm{W} 16^{\circ} 49^{\prime} 14^{\prime \prime} \text {, } \\
700 \mathrm{~m} \text {; laurisilva, 2003, Stüben }\end{array}$ & $\begin{array}{l}\text { ZFMK-DNA-JJ0510, } \\
\text { ZFMK-TIS-cE571ver }\end{array}$ & $\begin{array}{l}\text { GU988373 } \\
\text { GU987938 }\end{array}$ \\
\hline $\begin{array}{l}\text { Onyxacalles verrucosus } \\
\text { (Wollaston } 1863 \text { ) }\end{array}$ & $\begin{array}{l}\text { Spain: Canary Islands, Tenerife, NE La Laguna, Anaga } \\
\text { Mts. near Moquinal; N } 28^{\circ} 31^{\prime} 55^{\prime \prime} \mathrm{W} 16^{\circ} 17^{\prime} 24^{\prime \prime}, 840 \mathrm{~m} \text {; } \\
\text { laurisilva, 2008, Astrin and Stüben }\end{array}$ & $\begin{array}{l}\text { ZFMK-DNA-JJ0604, } \\
\text { ZFMK-TIS-cE677ver }\end{array}$ & $\begin{array}{l}\text { GU988433 } \\
\text { GU987995 }\end{array}$ \\
\hline $\begin{array}{l}\text { Onyxacalles verrucosus } \\
\text { (Wollaston 1863) }\end{array}$ & $\begin{array}{l}\text { Spain: Canary Islands, Tenerife, NE La Laguna, Anaga } \\
\text { Mts. near Chinobre; N } 28^{\circ} 33^{\prime} 21^{\prime \prime} \mathrm{W} 16^{\circ} 10^{\prime} 46^{\prime \prime}, 808 \mathrm{~m} \text {; } \\
\text { Laurus, Ixanthus viscosus, 2008, Astrin and Stüben }\end{array}$ & $\begin{array}{l}\text { ZFMK-DNA-JJ0605, } \\
\text { ZFMK-TIS-cE678ver }\end{array}$ & $\begin{array}{l}\text { GU988434 } \\
\text { GU987996 }\end{array}$ \\
\hline $\begin{array}{l}\text { Onyxacalles verrucosus } \\
\text { (Wollaston 1863) }\end{array}$ & $\begin{array}{l}\text { Spain: Canary Islands, Tenerife, SW Los Silos, Teno } \\
\text { Mts., Monte del Agua, Chupadero; N28 } 19^{\prime} 23^{\prime \prime} \\
\text { W16 } 49^{\prime} 12^{\prime \prime}, 940 \mathrm{~m} \text {; Laurus novocanariensis, 2008, } \\
\text { Astrin, Stüben, Behne and Floren }\end{array}$ & $\begin{array}{l}\text { ZFMK-DNA-JJ0776, } \\
\text { ZFMK-TIS-cE813ver }\end{array}$ & $\begin{array}{l}\text { GU988524 } \\
\text { GU988085 }\end{array}$ \\
\hline $\begin{array}{l}\text { Onyxacalles vilae sp.n. } \\
\text { (O. luigionii) }\end{array}$ & $\begin{array}{l}\text { France: Isère, } 2 \mathrm{~km} \text { SE Lans en Vercors, Montagne de } \\
\text { Lans; N } 45^{\circ} 06^{\prime} 45^{\prime \prime} \text { E } 05^{\circ} 36^{\prime} 21^{\prime \prime}, 1352 \mathrm{~m} \text {; Abies, Fagus, } \\
\text { Fraxinus, 2005, Stüben }\end{array}$ & $\begin{array}{l}\text { ZFMK-DNA-JJ0025, } \\
\text { ZFMK-TIS-cI0027lui }\end{array}$ & $\begin{array}{l}\text { EU286286 } \\
\text { EU286451 }\end{array}$ \\
\hline $\begin{array}{l}\text { Onyxacalles vilae sp.n. } \\
\text { (O. luigionii) }\end{array}$ & $\begin{array}{l}\text { Croatia: Dalmatian, } 8 \text { km E Karlobag, Velebit Mts., } \\
\text { Stupacinovo; N } 44^{\circ} 32^{\prime} 41^{\prime \prime} \text { E } 15^{\circ} 09^{\prime} 58^{\prime \prime}, 1049 \text { m; Fagus, } \\
\text { 2007, Stüben }\end{array}$ & $\begin{array}{l}\text { ZFMK-DNA-JJ0195, } \\
\text { ZFMK-TIS-cHR0339lui }\end{array}$ & $\begin{array}{l}\text { EU286348 } \\
\text { EU286512 }\end{array}$ \\
\hline
\end{tabular}

gaps. All of these were included into phylogenetic analysis. We implemented the GTR $+\mathrm{I}+\Gamma$ [29] model of nucleotide substitution for both genes in Bayesian MCMC analyses, run in MrBayes ver. 3.1.2 [30]. Only COI was included for the new sequences. We ran two independent replicates for 10 million generations per analysis (each with 1 cold chain and 3 chains of different temperature). Every 1'000th tree was sampled (20'000 trees retained). Negative log-likelihood score stabilisation was determined in a separate visualisation (in MS Excel). Accordingly, we retained 19.800 trees (after discarding burn-in), of which a 50\%-majority rule consensus tree was built, with posterior probabilities (Figure 1).

\section{Results and Discussion}

4.1. A Gestalt Switch-Changing the Way You See: Kyklioacalles pyrenaeus (Boheman, 1844). The species of Onyxacalles are characterised by a particularly long and slender rostrum which is at least 3-4 times as long as wide between the insertions of the antennae. A further conspicuous feature (not typical for western Palearctic Cryptorhynchinaeexcepting the species of the Macaronesian Islands) is the unusually long and slender (arachnoid) legs. The name of this genus refers to the hook-shaped tip of the aedeagus. No further species of the former accumulative genus Acalles or of other western Palearctic genera of the Cryptorhynchinae exhibit such a characteristic hook-shaped tip of the aedeagus (onyx; greek: hook, hook-shaped tool). Complex-sclerotised structures of the internal sac are absent or reduced to simple line- or bar-like structures. These structures have been significant, for instance, for the partly phylogeny-based classification and determination of the genera and species of Dichromacalles and Kyklioacalles. The strongly sclerotised median lobe exhibits either only unclear structures or none at all (see [31]).

As early as the beginning of the last century, in their ground-breaking revision of the western Palearctic species of Acalles s.l., A. and F. Solari placed the species A. pyrenaeus 
Boheman, 1844, A. henoni Bedel, 1888, A. croaticus Brisout, 1867, and A. luigionii Solari, 1907-although together with further species-into the same group (see IV. group; [32]). Together with initially 7 further species (among it the above species denominated by A. and F. Solari, as well as 3 further species from the Canary Islands), they were transferred into the new genus Onyxacalles [1, page 186], a genus that currently comprises 20 species.

However, Onyxacalles pyrenaeus is a polymorphic species, with regard to the outline of the aedeagus as well as the more or less ovally rounded elytra [1, page 188]. This is - among others-the reason why we could not ascertain definitively whether the subspecies Acalles pyrenaeus germanicus Letzner, 1882 (= Onyxacalles boehmei Košt'ál \& Holecová, 2001 [33] syn.) is really a junior synonym or not (even if the first author considers it a synonym; cf. [7, page 123]).

In addition to Onyxacalles portusveneris (Mayet, 1903) (see Figure 4), O. pyrenaeus has an exceptionally large distribution area (cf. [31]). This could explain the high genetic distances of the mitochondrial COI and 16S gene (e.g., France: Lans en Vercors-Austria: Merkersdorf, p-distances: $\mathrm{COI}=8,5 \%, 16 \mathrm{~S}=2,4 \%)$. This species can be found from the Pyrenees to the mountains of Western, Central, and Eastern Europe to the Carpathians and can be beaten from the branches of different conifers, especially larch, but can also be sifted under deciduous trees (e.g., Fagus).

This does not coincide with the other Onyxacalles species, which live on different deciduous trees and never prefer conifers. Apart from these ecological conditions, we can establish that the above-mentioned differences to the other genera of the western Palearctic Cryptorhynchinae point to a closer relationship to the species of Onyxacalles, a view with which most authors concur (e.g., [33]).

In any case, the taxonomists did not pay attention to the distinct ecology and-in comparison with the other species of Onyxacalles - the clearly narrower aedeagus (cf. Figure 6(k) versus Figures 9(a)-9(1)). Are these peripheral characteristics? And in which genus of Cryptorhynchinae is it possible to place this species (without the need for a monotypic genus)? The first author had never imagined assigning this species to the genus Kyklioacalles Stüben, 1999. There was no reason for such a review, not even an "initial suspicion" (see below).

However, this presumed "Onyxacalles" species appears deeply nested within the genus Kyklioacalles in the dendrogram [34, Figure 1]. This inclusion within Kyklioacalles is maximally supported. Furthermore, it is obvious that Acalles pyrenaeus forms a clade with $K$. fausti (Meyer, 1896), K. reginae Stüben, 2003, K. saccoi (Colonnelli, 1973) [35], and K. reinosae (H. Brisout de Barneville, 1867) [10, 34]. Two species, $K$. reginae and $K$. reinosae, are distinguished from the other species by the completely different habitus, but chiefly belong and were allocated early on to the $K$. fausti group "only" on the basis of the endophallus. This allocation has been shown to be justified by molecular data [34].

But what would have happened if Kyklioacalles had not been defined initially on the basis of the cyclical structure of the endophallus, as the name implies [36], and as an immediate consequence, Acalles pyrenaeus would not have been eliminated? Putting it the other way round: what if the similarity between this species Kyklioacalles saccoi and Kyklioacalles fausti had been considered in a habitus-tohabitus comparison? That is not only a simple, retrospective, and dispensable "what-if" question, because in this case one brings the fact to mind that $A$. pyrenaeus must now be coercively placed among the Kyklioacalles species, Figures 8(f)$8(\mathrm{~h})$.

The definition especially of a higher taxon is an arbitrary supposition. As taxonomists we always operate with constructs and as morphologists we find characteristics that are prominent to our eyes (and sometimes "we like to see"). It must be admitted that extremely rarely do we look for homologies, which in theory constitute the best criterion (e.g., [37]), but are in practice often difficult to find when dealing with cryptic and similar-looking species.

This change of mind and perception is similar to a Gestalt switch [22], a figure spinning in two directions: the contour line of the species is the same, but the species is not (as in the Gestalt psychology, the vase-face and duckrabbit illusions). We cannot explain this Gestalt switch based on morphological research alone, and we cannot build and establish it within this framework. But we know the cause for this inconspicuous paradigm change: only extrinsic evidence from DNA analysis has opened the morphologist's eyes in this case- and this in the true sense of the word.

4.2. Integrative Taxonomy: Changing the Way You Look for Species. Integrative taxonomy sounds like an accumulation of different disciplines: morphology, molecular biology, ecology, ethology, and biogeography deliver the ingredients. But this is not invariably the case, and not so simple. It is a more eventful, reciprocal exchange of evidence, which an initial suspicion either confirms or rejects (comparable to an unsolved criminal case).

An initial morphological suspicion was already available when we discovered the new species Onyxacalles nuraghi (O. henoni group) in the humid Quercus/Acer forest in the mountains of Marghine on Sardinia. The differences from the well-known species of this group are obvious and easy to assemble (see below differential diagnosis of $O$. nuraghi). In the case of Onyxacalles vilae sp. n. (O. luigionii group) from the Velebit Mountains (Croatia), it was morphologically more ambiguous, but in case of the third species Onyxacalles torre sp.n. (O. henoni group) from Corsica, the specimens of the current type series remained completely unnoticed in the collection for years.

But the molecular results in view of these three (resp. four) species are obvious (see also Figures 1-4). With maximal support, $O$. vilae is widely separated in the tree topology from $O$. nuraghi and $O$. torre (O. henoni group; Figure 1). Together with Onyxacalles sp. 1, O. vilae groups with $O$. luigionii (Onyxacalles sp. 1 as sister taxon of the latter), but all these species are separated by considerable p-distances ( $>9 \%$ COI and $>4 \% 16 \mathrm{~S}$; Figure 3 ). Interestingly, the O. vilae specimen from Croatia shares the haplotype of the French specimen. O. torre and O. nuraghi are genetically closer to each other (1,8\% 16S; Figure 2), but not sister species. 


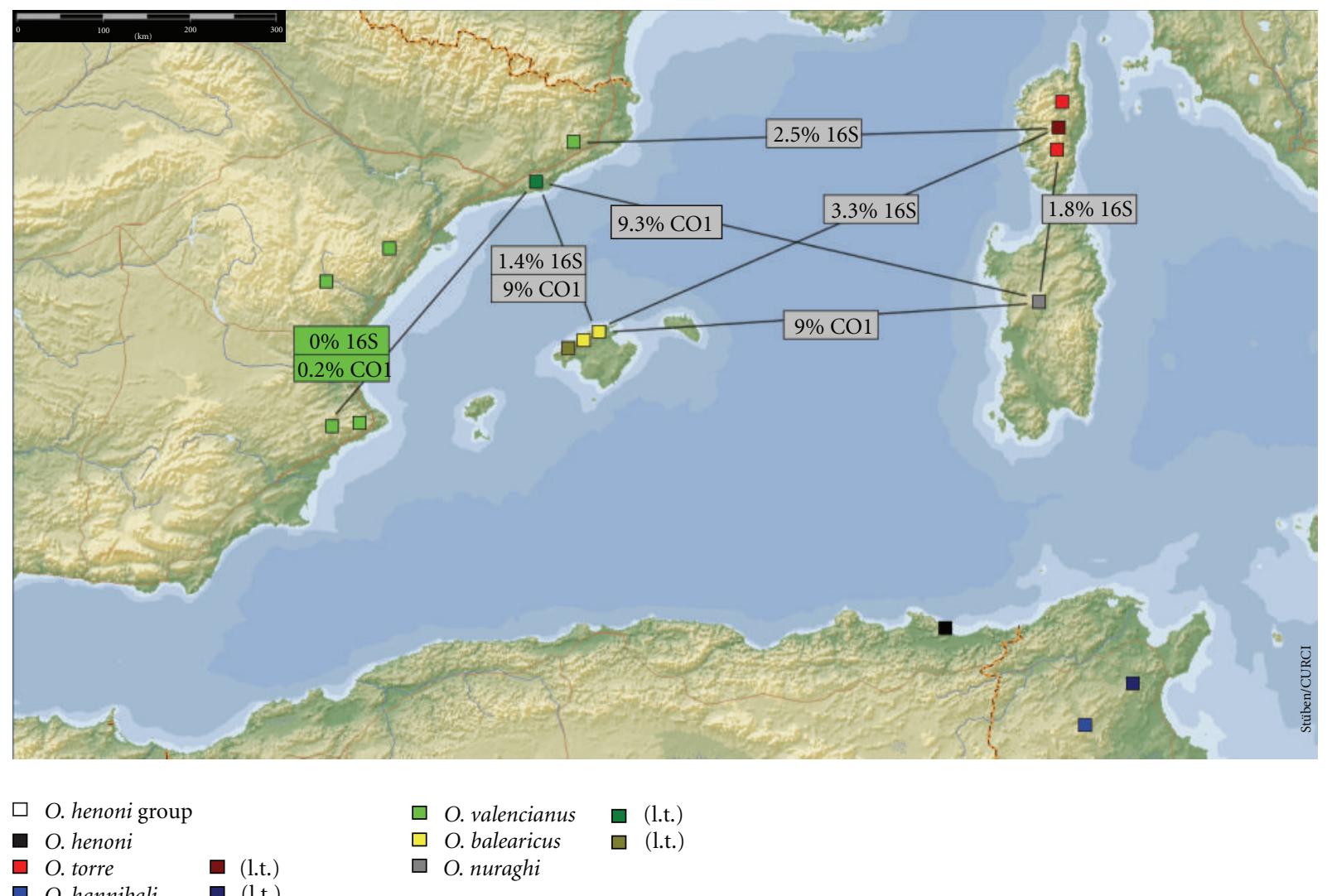

FIGURE 2: Distribution of related species of the West Mediterranean species of the $\boldsymbol{O}$. henoni group (without O. croaticus, Eastern Europe) with values of COI and $16 \mathrm{~S}$ p-distances.

The unambiguous molecular evidence (cf. Figures 2 and 3) prompts the morphological reinvestigation of the material and forces the morphologist to look for new characters (see below "taxonomy")—if they exist!

On the one hand-in the case of Onyxacalles species-we currently give more attention to the apex of the rostrum and its punctures, or of the more or less curved and hook-shaped apex of the aedeagus. Vice versa, we see in the conspicuous and serially placed elytral bristles and its erected tufts on the intervals "seductive anthropomorphisms" and analyses of yesteryears. The perception does not become more precise, and the tuning is not finer adjusted-rather the way we view characters has changed: the perspective regarding criterion and weighting has undergone a shift influenced by extrinsic data.

On the other hand, however, the few specimens in the immediate vicinity of the French village Sospel (Maritime Alps) attest that it does not succeed in all cases (see: Figure 1: Onyxacalles sp. 1). The high p-distances of the mitochondrial COI and $16 \mathrm{~S}$ genes of these specimens compared to the Middle and South Italian populations of $O$. luigionii and to $O$. vilae from the Velebit Mountains and the French Isère indicate the plausibility of a new species (cf. Figure 3 ). However, morphological differences could not be diagnosed. More findings and larger series of comparison material should establish a clearer picture in future. Therefore, it is premature to postulate a cryptic species and contrast it in a differential diagnosis by nothing more than molecular characters at this stage. Furthermore, this action would have the direct disadvantage that all previous existing records from the French and Italian Maritime Alps could not be allocated to the above-mentioned species. Nevertheless, this could shift in the future, should in depth "DNA barcodes" become available for all applied entomological disciplines.

The first step in this development has already been taken: the Molecular Weevil Identification-Project (ZFMK, CURCI), which is going to establish a molecular (DNA barcodes) and photographic database (stacked images) as well as the highly important associated reference collections for European Curculionoidae (ca. 6000 species). Only by meticulous cross-vouchering can misidentifications be corrected, and molecular results can be linked to the more than 250 years history of entomology. Integrative taxonomy is not just an accumulative or encyclopaedic "furthering of knowledge," but rather-as in this case-an interactive process in an interdisciplinary dialogue.

\section{Taxonomy}

Family: Curculionidae Latreille, 1802.

Subfamily: Cryptorhynchinae Schoenherr, 1825. 


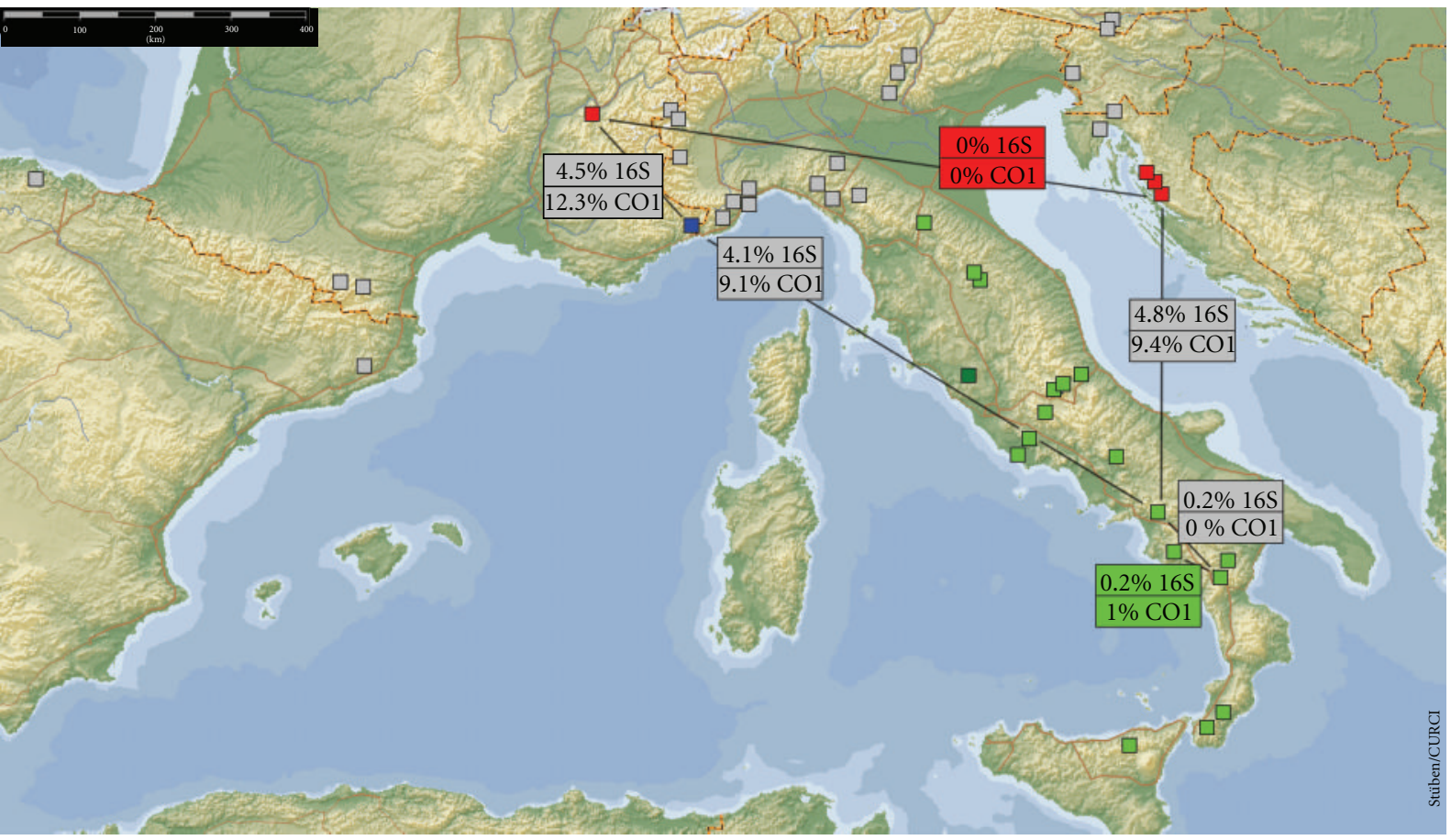
$\square$ O. luigionii group
$\square$ O. luigionii
(1.t.)
O. vilae
ㅇ.sp. 1

FIGURE 3: Distribution of related species of the $\boldsymbol{O}$. luigionii group in the Mediterranean area with values of COI and $16 \mathrm{~S}$ p-distances.

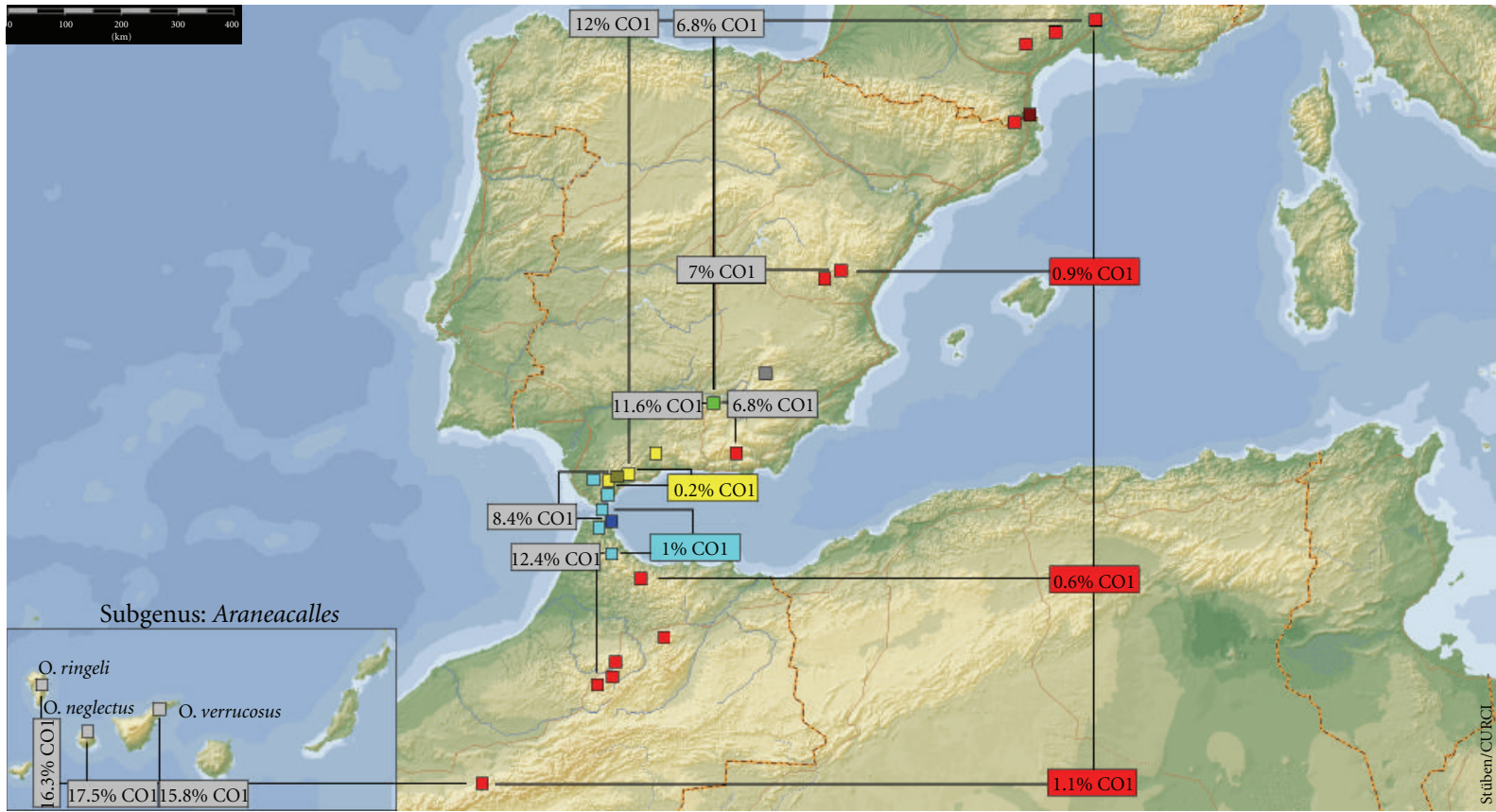
$\square$ O. portusveneris group
$\square$ O. portusveneris
$\square$ O. maginaensis
$\square$ O. gibraltarensis
口(l.t.)
$\square$ O. seguraensis
$\square$ O.bermejaensis
$\square$ (1.t.)

FIgUre 4: Distribution of related species of the O. portusveneris group in the Mediterranean area with values of COI p-distance (658 bp). 
Genus: Onyxacalles Stüben, 1999, Type species: Acalles luigionii A. and F. Solari, 1907 (L.t.: Central Italy).

\subsection{New Species of the Onyxacalles henoni Group}

\subsubsection{Onyxacalles nuraghi Stüben sp. n.}

(Figures 5(a)-5(c), 5(i), 6(1)).

\section{Type Material}

Holotype (10 $)$. Italy: Sardinia, Macomer, Lei, N40 $19^{\prime} 54^{\prime \prime}$ E08 53'49", $1020 \mathrm{~m}$, Quercus, Acer monspesulanus, 4.10.2010, leg. Stüben-27-, coll. CURCULIO-Institut, D-Mönchengladbach.

Paratypes (10 ). Data as for holotype, coll. Stüben; $40^{7}$, 1 : Italy: Sardinia, Macomer, Lei, N40 $19^{\prime} 17^{\prime \prime} \mathrm{E} 08^{\circ} 53^{\prime} 52^{\prime \prime}$, $586 \mathrm{~m}$, Quercus ilex, 7.10.2010, leg. Stüben-33-, coll. Stüben, CURCULIO-Institut, D-Mönchengladbach (1\%), coll ZFMK, $10^{x}$ : ZFMK-DNA-JJ1097, ZFMK-TIS-cIT1097.

DNAtype $\left(1 \sigma^{x}\right)$. Data as for holotype, coll. ZFMK: ZFMKDNA-JJ1090, ZFMK-TIS-cIT1090; GenBank Acc. no COI: JN121399, 16S: JN642097.

Differential Diagnosis. The new species from the southfacing slope of the Chain of Marghine (Italy: Sardinia) belongs-from a morphological and molecular perspectiveto the Onyxacalles henoni group and should be compared with the most closely related species from Majorca (Spain): Onyxacalles balearicus Stüben, 2005.

\section{Onyxacalles nuraghi}

(1) Disc of pronotum with a channel from the base towards the flat sector in front of the fore-margin; with tufts of bristles on both sides of the channel (Figure 5(i)).

(2) Bristles on the elytral intervals at least $2 \mathrm{x}$ as long as wide; shaping tufts with big gaps; their distances range from $3 x$ the length of bristles.

(3) Elytra of male with parallel sides in the middle sector (dorsal view); contour line of elytra forms almost a semicircle in lateral view (Figure 5(a)).

(4) Apex ("hook") of the aedeagus (in ventral view) smaller (Figure 5(c)).

\section{Onyxacalles balearicus}

$\left(1^{*}\right)$ Disc of prontoum without a channel and without tufts of bristles (Figure 5(h)).

$\left(2^{*}\right)$ The free-standing bristles on the intervals shorter, $1.3 \mathrm{x}$ as long as wide; placed in a single row, not forming tufts.

(3*) Elytra of male broader and stronger (short ovally) rounded (slightly "egg shaped"); contour line of elytra flatter or slightly rounded behind the base in lateral view (Figure 5(g)).

$\left(4^{*}\right)$ Apex (hook) of the aedeagus (in ventral view) broader (Figure 5(j)).

The new species from Sardinia is different from Onyxacalles henoni (Bedel, 1888) [38] (Algeria: Mt. Edough, loc. typ.), with which it has the tufts of bristles on the elytral intervals in common, by (1) darker elytral integument (Figure 5(a) versus 5(k)), (2) finer and longer white bristles on the femora, and (3) longer apex ("hook") of the aedeagus (lateral view, see Figure 5(c) versus 5(n)). It can be distinguished from Onyxacalles valencianus Germann, 2005 from the Spanish mainland (Barcelona: Villarana, loc. typ.) by (1) elytral tufts of bristles (versus single bristles), (2) deep channel of the pronotum (versus without channel), and (3) longer apex of aedeagus (lateral view, see Figure 5(c) versus $9(\mathrm{~h}))$.

For a comparison with all other species see below the "Key to the species, of Onyxacalles Stüben, 1999".

\section{Description}

Length. 2.60-3.40 $\mathrm{mm}$ (without rostrum).

Head and Rostrum. Eyes large; rounded ovally towards front and acuminate towards underside of rostrum; frons between eyes more slender than the base of rostrum; rostrum reddish brown, closely covered with white scales at the base; rostrum of male reaching 3/4 length of pronotum and finely punctuated towards apex; rostrum of females reaching $4 / 5$ length of pronotum, slender, shiny, and even more finely punctuated. The last three funicles of antennae short ovally rounded; the first two funicles elongated; the club clearly separated from funicles.

Pronotum. Widest at the end of the first third of the pronotum (holotype: $1.17 \mathrm{x}$ as wide as long); well rounded laterally towards the fore-margin and the base; with a deep depression at the sides directly behind the fore-margin; disk of pronotum strongly arced, with a channel in the middle from the base towards the flat sector in front of the foremargin. The integument is rich in contrast consisting of round black scales on the disk and oval, white/brown scales on the flanks of the pronotum. Elongated and black bristles in an upright position in the middle of the disk on both sides of the channel; with a similar, but white tuft of bristles on each side of the pronotum; the deep punctures always covered with scales.

Elytra. Oblong (holotype: $1.29 \mathrm{x}$ as long as wide); widest in the middle and there with nearly parallel sides; only slightly rounded directly in front of the base; short ovally rounded towards the apex. Contour line of elytra strongly arced, almost forming a semi circle. The shiny and predominantly dark brown integument with a beige/white crescent-shaped fascia in front of the base and on the elytral slope. Bristles on the first and third interval (excluding the suture stripe) 


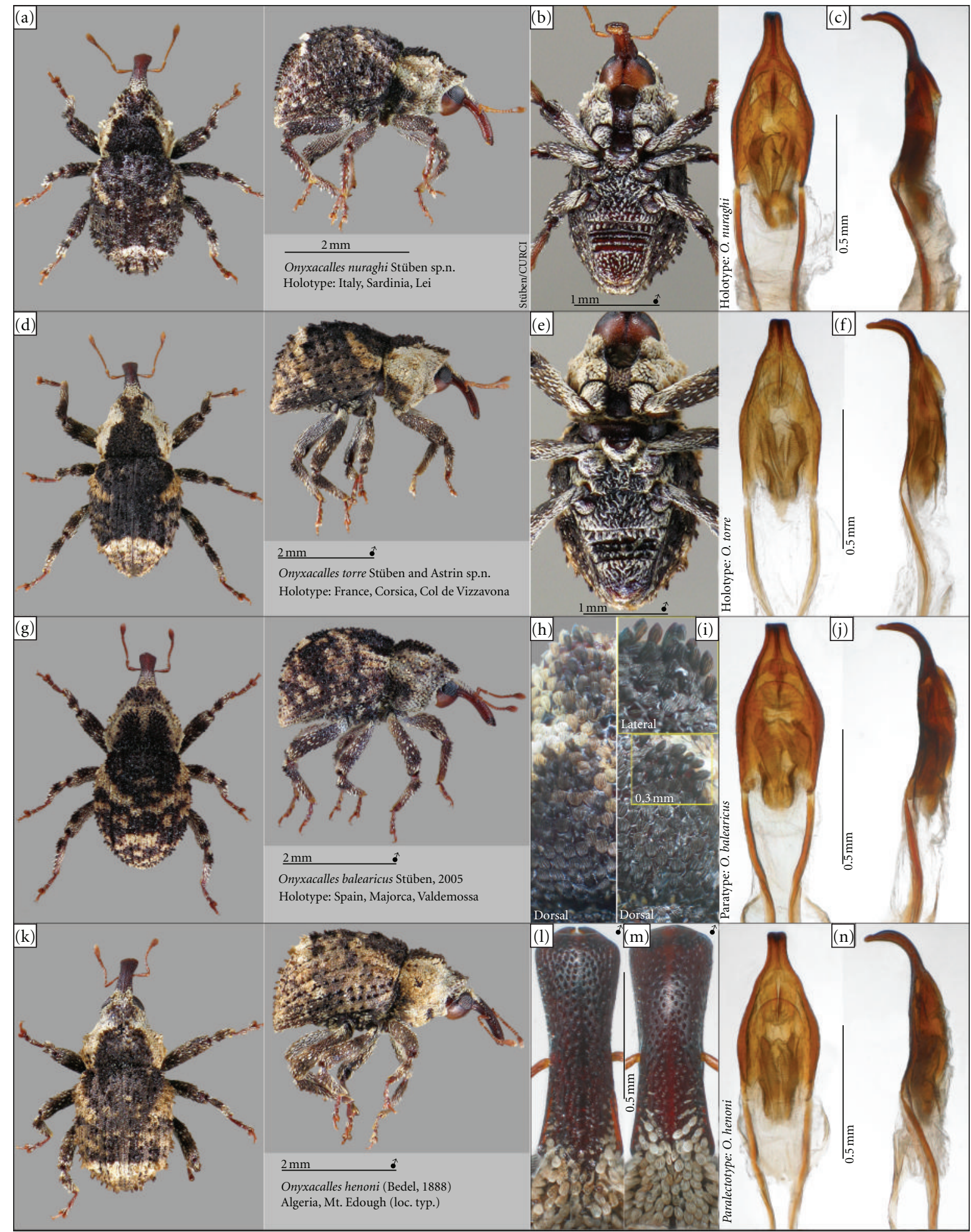

Figure 5: (a)-(c) O. nuraghi sp.n.-habitus (dor./lat./ven.), aedeagus (ven./lat.). (d-f) O. torre sp. n.- habitus (dor./lat./ven.), aedeagus (ven./lat.); (g, j) O. balearicus—habitus (dor./lat.), aedeagus (ven./lat.); (k, n) O. henoni—habitus (dor./lat.), aedeagus (ven./lat.). By comparison, bristle of pronotum-O. balearicus (h) versus O. nuraghi (i); rostrum-O. henoni (l) versus O. torre (m). 

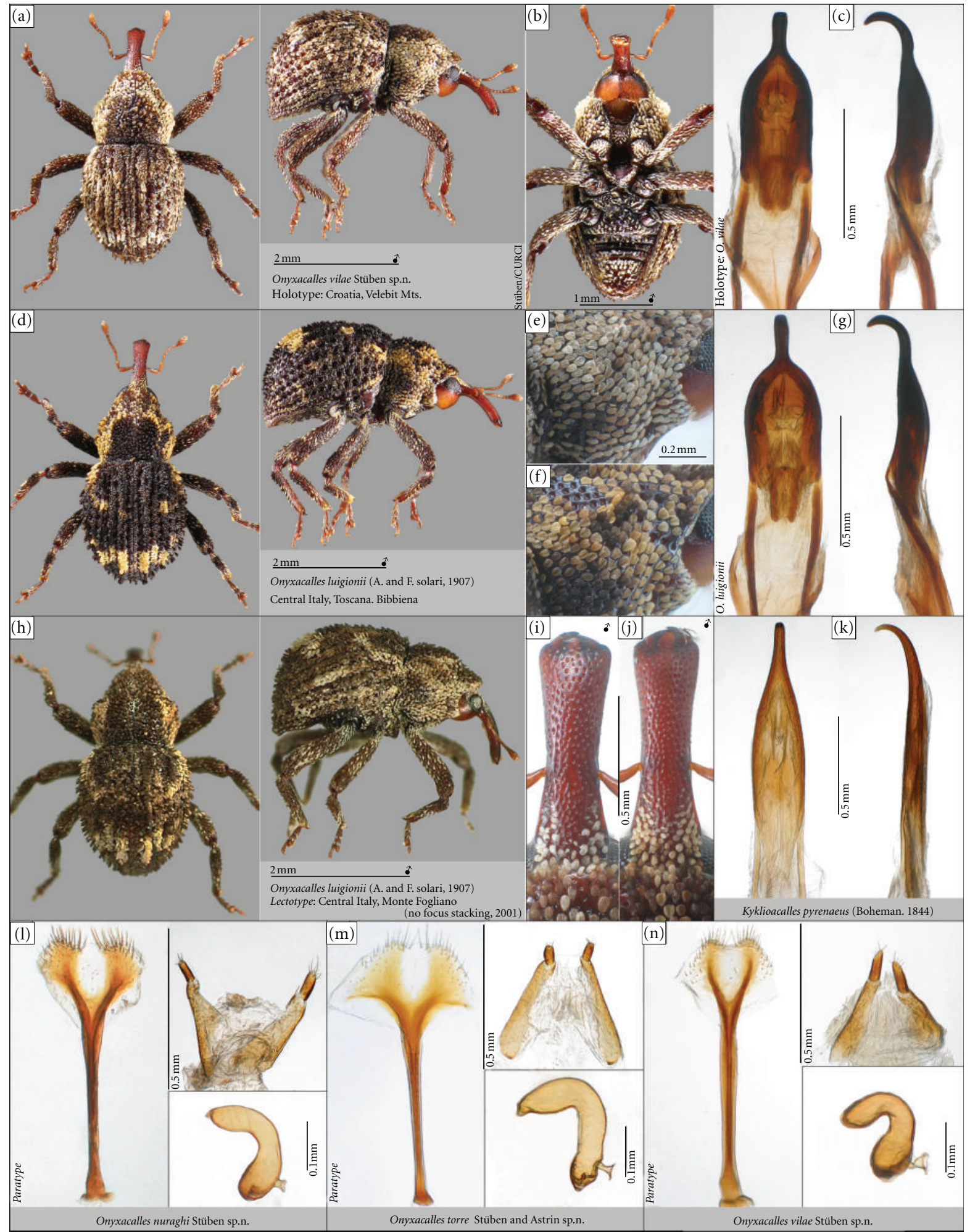

Figure 6: (a-c) O. vilae sp. n.-habitus (dor./lat./ven.), aedeagus (ven./lat.); (d, g) O. luigionii-habitus (dor./lat.), aedeagus (ven./lat.); (h) O. luigionii (lectotype)—habitus (dor./lat.); (k) Kyklioacalles pyrenaeus_aedeagus (ven./lat.); (l-n) female genital (spiculum ventrale, ovipositor, spermatheca) of O. nuraghi sp.n. (1), O. torre (m) and O. vilae (n). By comparison, bristle of pronotum (lat.) -O. vilae (e) versus O. luigionii (f); rostrum-O. vilae (j) versus O. luigionii (j). 
at most $2 \mathrm{x}$ longer than wide, forming flattened tufts, which have big gaps between them (their distances range from three times the length of bristle); bristles sparse on the second and fourth intervals; striae on the disc and at the sides of elytra clearly narrower than intervals, punctures oblong.

Legs. Long; the marginal front femora reach the base of the rostrum; the hind femora reach the end of the elytral apex. They are covered with predominantly dark brown scales; tibia with long, white, and laterally protruding bristles.

Venter. The 2nd strip-type sternite only a little bit longer than the 3 rd, but not longer than sternites 3 and 4 together. 1st sternite of male with a broad depression (Figure 5(b)).

Female Genital. See Figure 6(1).

Aedeagus. Apex ("hook") of the aedeagus (in ventral view) small, see Figure 5(c).

Etymology. The species name refers to the Nuragic civilization of Sardinia, lasting from the Bronze Age (18th century $\mathrm{BC})$ to the 2 nd century $\mathrm{AD}$.

Ecology. Onyxacalles nuraghi was discovered by the first author on Sardinia near Lei (Macomer) in the mountains of Marghine in 2010. The seven specimens were shifted under Quercus and Acer between 500 and $1000 \mathrm{~m}$ above sea level.

Distribution. This species is so far only known from the Chain of Marghine, Figure 2.

\subsubsection{Onyxacalles torre Stüben and Astrin sp. n.}

(Figures 5(d)-5(f), 5(m), and 6(m)).

\section{Type Material}

Holotype (1 $\left.10^{\pi}\right)$. France, Corsica (Haute-Corse): Col de Vizzavona, $22 \mathrm{~km} \mathrm{~S}$ Corte, $1100 \mathrm{~m}, 8.10 .2001,42^{\circ} 06^{\prime} 45^{\prime \prime} \mathrm{N}$ $09^{\circ} 06^{\prime} 49^{\prime \prime}$ E, Fagus (sift), leg. Stüben-4-, coll. CURCULIOInstitut, D-Mönchengladbach.

Paratypes $\left(80^{\prime}, 99\right)$. Data as for holotype, coll. Stüben, CURCULIO-Institut, D-Mönchengladbach (19), Zoologisches Forschungsmuseum Alexander Koenig, D-Bonn (10 1ㅇ).

DNAtype (19). Data as for holotype, coll. ZFMK: ZFMKDNA-JJ0882, ZFMK-TIS-cF479; GenBank Acc. no 16S: GU988592.

Further Material $\left(10^{7}\right)$. France, Corsica (Haute-Corse): Caporalino $10 \mathrm{~km} \mathrm{~N}$ Corte, $350 \mathrm{~m}, 7.10 .2001,42^{\circ} 23^{\prime} 08^{\prime \prime} \mathrm{N}$ $09^{\circ} 11^{\prime} 37^{\prime \prime} \mathrm{E}$, Alnus, Fraxinus, Quercus (sift), leg. Stüben-2-, coll. Stüben; $20^{\top}$ : France, Corsica (Haute-Corse): Tattone,
$18 \mathrm{~km}$ S Corte, $750 \mathrm{~m}, 8.10 .2001,42^{\circ} 09^{\prime} 21^{\prime \prime} \mathrm{N} 09^{\circ} 09^{\prime} 43^{\prime \prime} \mathrm{E}$, Castanea (sift), leg. Stüben-3-, coll. Stüben; 10': France, Corsica (Corse-du-Sud): Radicale, $20 \mathrm{~km}$ E Ajaccio, $400 \mathrm{~m}$, 9.10.2001, $41^{\circ} 55^{\prime} 31^{\prime \prime} \mathrm{N} 08^{\circ} 58^{\prime} 10^{\prime \prime} \mathrm{E}, 9 . \mathrm{X} .2001$, Quercus ilex (sift), leg. Stüben-9-, coll. Stüben; 20': France, Corsica (Corse-du-Sud): Cozzano $2 \mathrm{~km}$ NE Zicavo, $750 \mathrm{~m}$, 10.10.2001, $41^{\circ} 55^{\prime} 31^{\prime \prime} \mathrm{N} 09^{\circ} 08^{\prime} 31^{\prime \prime} \mathrm{E}$, Castanea (sift), leg.

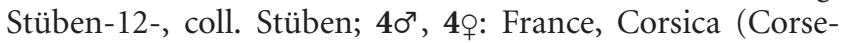
du-Sud): Coll de La Vaccia N, 9,5 km S Zicavo, $1150 \mathrm{~m}$, 10.10.2001, $41^{\circ} 49^{\prime} 19^{\prime \prime} \mathrm{N} \quad 09^{\circ} 05^{\prime} 04^{\prime \prime} \mathrm{E}$, Fagus (sift), leg. Stüben-14-, coll. Stüben.

Differential Diagnosis. The new species from Corsica (France) belongs-morphologically and molecularly-to the Onyxacalles henoni group and is distinguished from Onyxacalles henoni (Bedel, 1888) [38] from Algeria (Mt. Edough, loc. typ.) by the following characteristics.

\section{Onyxacalles torre}

(1) Rostrum finely punctuated towards apex (Figure 5(m)).

(2) Scales of the elytra predominantly dark brown or black (Figure 5(d)).

(3) Apex ("hook") of the aedeagus broader in ventral view and strongly curved (nearly rectangular) in lateral view (Figure 5(f)).

\section{Onyxacalles henoni}

$\left(1^{*}\right)$ Rostrum coarsely and densely punctuated towards apex (Figure 5(l)).

$\left(2^{*}\right)$ Scales of the elytra predominantly bright: white, beige, or brown (Figure 5(k)).

(3*) Apex ("hook") of the aedeagus smaller in ventral view and not so strongly curved in lateral view (Figure 5(n)).

The new species from Corsica can be distinguished from Onyxacalles nuraghi from Sardinia (see above) by (1) contour line of elytra behind the base flatter (in lateral view, Figure 5(d) versus 5(a)), (2) elytra more egg-shaped towards the apex (Figure 5(d) versus 5(a)), and (3) apex ("hook") of the aedeagus (in ventral view) shorter and wider (Figure 5(f) versus $5(\mathrm{c})$ ). For a comparison with all other species, see the "Key to the species of Onyxacalles Stüben, 1999" below.

\section{Description}

Length. 3.00-4.00 mm (without rostrum).

Head and Rostrum. Eyes large; rounded towards front and acuminate towards underside of rostrum; frons between eyes as wide as the base of rostrum; rostrum reddish brown, closely covered with white scales at the base; rostrum of male reaching $2 / 3$ length of pronotum and finely punctuated towards apex (Figure $5(\mathrm{~m})$ ); rostrum of females reaching $3 / 4$ 
length of pronotum, slender, shiny, and even more finely punctuated. The last three funicles of the antennae short ovally rounded, the fourth $1.5 \mathrm{x}$, the third $2 \mathrm{x}$, the second $4.5 \mathrm{x}$, and the first conical funicle $2 \mathrm{x}$ longer than wide; the elongated club clearly separated from funicles.

Pronotum. Widest at the end of the first third of the pronotum (holotype: $1.17 \mathrm{x}$ as wide as long); strongly rounded laterally towards the fore-margin and the base; with a depression at the sides directly behind the fore-margin; disk of pronotum arced, in the middle sometimes with a slight channel-like depression in front of the base. The integument is rich in contrast consisting of round black and dark-brown scales on the disk and in front of the fore-margin, and more or less oval, white scales on the flanks of the pronotum. In the middle of the disk on both sides of the flat depression with elongated, studded, and black bristles in an upright position; with similar placed, but shorter and white bristles on each side of the pronotum; the punctures always covered with scales.

Elytra. Oblong (holotype: $1.31 \mathrm{x}$ as long as wide); widest in front of the middle, here with parallel sides or slightly eggshaped towards the apex; strongly curved in front of the base. Contour line of elytra flatter behind the base in lateral view, the contour line of the elytral slope forming an arc towards the apex. The shiny and predominantly dark brown or black integument with a beige/white crescent-shaped fascia in front of the base and on the elytral slope; sometimes the whole apex can be light brown. Bristles on first and third interval (excluding the suture stripe) $1.5 x$ longer than wide, flattened, and shaping tufts; their distances range from the $2 x$ length of bristles. Bristles sparse on the second and fourth intervals; striae on the disc and at the sides of elytra are small strips, clearly narrower than the intervals, punctures on the disc oblong, round at the sides.

Legs. Long; the marginal front femora reach the base of the rostrum, and the hind femora reach the end of the elytral apex. They are covered with predominantly dark brown and elongated scales; tibia with white/brown and laterally protruding bristles forming fasciae.

Venter. The 2nd strip-type sternite only a little bit longer than the $3 \mathrm{rd}$, but not longer than sternites 3 and 4 together. 1st sternite of male with a broad depression (Figure 5(e)).

\section{Female Genital. See Figure 6(m).}

Aedeagus. Apex (hook) of the aedeagus broad in ventral view and strongly curved (nearly rectangular) in lateral view; see Figure 5(f).

Etymology. The species name refers to the Torrean civilization in Corsica during the second millenium BC. The characteristic building of this culture is the "Torre" (tower), the Corsican counterpart of the Sardinian "Nuraghe."
Ecology. Onyxacalles torre was sifted by the first author in the mountains of Corsica and is a nocturnal inhabitant of the dark and shady forests like all other Onyxacalles.

Distribution. This species is so far only known from Corsica (France); Figure 2.

\subsection{New Species of the Onyxacalles luigionii Group}

\subsubsection{Onyxacalles vilae Stüben sp. $\mathrm{n}$.}

(Figures 6(a)-6(c), 6(e), 6(i), and 6(n)).

\section{Type Material}

Holotype $\left(10^{7}\right)$. Croatia: $20 \mathrm{~km} \mathrm{~S} \mathrm{Krasno} \mathrm{Polje,} \mathrm{Northern}$

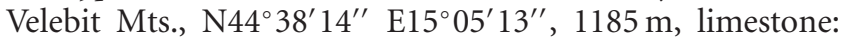
Fagus, 26.7.2004, leg. Stüben-10-, coll. CURCULIO-Institut, D-Mönchengladbach.

Paratypes $\left(10^{7}, 20\right)$. Data as for holotype, coll. Stüben, CURCULIO-Institut, D-Mönchengladbach (1o), Zoologisches Forschungsmuseum Alexander Koenig, D-Bonn (10); 1o: Croatia: Krasno Polje, Northern Velebit Mts., N $44^{\circ} 49^{\prime} 40^{\prime \prime} \mathrm{E} 15^{\circ} 01^{\prime} 49^{\prime \prime}, 838 \mathrm{~m}$, limestone: Fagus, Quercus, 25.7.2004 leg. Stüben-7-, coll. Stüben; $10^{7}$ : Croatia: $5 \mathrm{~km}$ W Krasno Polje, Northern Nord-Velebit Mts., N44 $48^{\prime} 49^{\prime \prime}$ E14 $58^{\prime} 36^{\prime \prime}, 1534 \mathrm{~m}$, limestone: Fagus, 26.7.2004, leg. Stüben-8-, coll. Stüben; 1o: Croatia: $6 \mathrm{~km}$ W Krasno Polje, Northern Velebit Mts., N44 $48^{\prime} 56^{\prime \prime}$ E14 $58^{\prime} 08^{\prime \prime}, 1494 \mathrm{~m}$, limestone: Fagus, 26.7.2004, leg. Stüben-9-, coll. Stüben; 10 ${ }^{7}$ : Croatia: $12 \mathrm{~km} \mathrm{~S} \mathrm{Krasno} \mathrm{Polje;} \mathrm{Northern} \mathrm{Velebit}$ Mts., N44 $43^{\prime} 00^{\prime \prime}$ E14 $4^{\circ} 59^{\prime} 42^{\prime \prime}, 1414 \mathrm{~m}$, limestone: Fagus, 27.7.2004, leg. Stüben-14-, coll. Stüben.

DNAtype $\left(10^{\top}\right)$. Croatia: $8 \mathrm{~km}$ E Karlobag, Velebit Mts.,

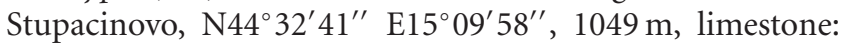
Fagus, 14.07.2007, leg. Stüben-27-, coll. ZFMK: ZFMKDNA-JJ0195, ZFMK-TIS-cHR339; GenBank Acc. no COI: EU286512, 16S: EU286348.

Further Material (90', 7\%). France, Isère, $2 \mathrm{~km}$ SE Lans en Vercors, Montagne de Lans, $45^{\circ} 06^{\prime} 40^{\prime \prime} \mathrm{N} 05^{\circ} 36^{\prime} 25^{\prime \prime} \mathrm{E}$, 1391 m, 23.7.2011, Kalk: Fagus (beaten), leg. Stüben-2-, coll. Stüben; 30 3 3 : France, Isère, NW Lans en Vercors: near Autrars, Parc Regional du Vercors, $45^{\circ} 14^{\prime} 12^{\prime \prime} \mathrm{N} 05^{\circ} 34^{\prime} 58^{\prime \prime} \mathrm{E}$, 1370 m, 23.7.2011, Kalk: Fagus (beaten), leg. Stüben-5-, coll. Stüben.

Differential Diagnosis. The new species from Croatia (Velebit Mts., loc. typ.) belongs-morphologically and molecularlyto the Onyxacalles luigionii group and is distinguished from Onyxacalles luigionii (A. \& F. Solari, 1907) [32] from Central Italy (Monte Fogliano, loc. typ.) by the following characteristics.

Onyxacalles vilae

(1) Rostrum of the male broader, $2.8 \mathrm{x}$ as long as wide (as measured by apex); punctures not so densely packed 
in front of the apex (separated by flat intervals) Figure 6(i).

(2) Bristles of the low-contrast elytra on first and third intervals (excluding the sutural stripe) longer and more slender; their distance is larger (Figure 6(a)).

(3) Scales of the white fascia at the sides of the pronotum (behind the base) predominantly oblong (Figure 6(e)).

(4) Median lobe of aedeagus smaller, $1.64 \mathrm{x}$ as long as wide; apex ("hook") a little bit shorter (in ventral view), flatter, and not so strongly curved in lateral view (Figure 6(c)).

\section{Onyxacalles luigionii}

( $\left.1^{*}\right)$ Rostrum of the male more slender, $3.1 \mathrm{x}$ as long as wide; punctures mainly dense towards the apex (only separated by small ridges) Figure 6(j).

$\left(2^{*}\right)$ Bristles of high-contrast elytra on the on first and third intervals shorter, broader (towards the apex of bristle), and more dense, clearly visible on the white fascia of the elytral slope (Figures 6(d) and 6(h)).

$\left(3^{*}\right)$ Scales of the white fascia at the sides of the pronotum (behind the base) predominantly round (Figure 6(f)).

$\left(4^{*}\right)$ Median lobe of aedeagus broader, $1.93 \mathrm{x}$ as long as wide; apex ("hook") of the aedeagus longer (in ventral view), strongly (nearly rectangular) curved in lateral view. Internal structure (endophallus) of the sac different (Figure 6(g)).

For a comparison with all other species see below the "Key to the species of Onyxacalles Stüben, 1999.'

\section{Description}

Length. 2.40-3.20 $\mathrm{mm}$ (without rostrum).

Head and Rostrum. Eyes large; rounded ovally towards front and acuminate towards underside of rostrum; frons between eyes as wide as the base of rostrum; rostrum reddish brown, closely covered with white and oval scales at the base; rostrum of male $2.8 \mathrm{x}$ as long as wide (as measured by apex) and finely punctuated towards apex, here separated by flat intervals (Figure 6(i)); rostrum of female clearly longer, slender, shiny, and even more finely punctuated (without punctures in front of the apex). The last funicles of antennae nearly trapezoidal, the funicles 4-6 short oval, the third funicle $1.3 \mathrm{x}$, the second $3 \mathrm{x}$, and the first conical funicle $1.5 \mathrm{x}$ longer than wide; the elongated club not clearly separated from the 7 th trapezoid funicle.

Pronotum. Widest at the end of the first third of the pronotum (holotype: $1.12 \mathrm{x}$ as wide as long); well rounded laterally towards the fore-margin and the base; with a slight depression at the sides directly behind the fore-margin; disk of pronotum arced, without a channel or a flat depression in the middle. The integument not so rich in contrast, consisting of round, brown scales on the disk and off-white, predominantly oblong scales at the sides of the pronotum (behind the base, Figure 6(e)). In the middle of the disk with elongated brown bristles in an upright position; with a similar, but white tuft of bristles on each side of the pronotum; the deep and dense punctures covered with scales.

Elytra. Short oval (holotype: 1.19x as long as wide); widest at the end of the first fourth in front of the elytral base; here laterally strongly rounded directly in front of the base; ovally rounded towards the apex. Contour-line of elytra flatter behind the base in lateral view, the contour line of the elytral slope forming a circular arc towards the apex. Bristles of the low-contrast elytra on first and third intervals (excluding the sutural stripe) slender, $2 \mathrm{x}-3 \mathrm{x}$ as long as wide, their distance reaching the double length of bristle, and forming tufts only in front of the base and on the elytral slope; the uprightly protruding bristles on the second and fourth intervals have wider gaps between them (their distances range from threeto fourfold length of bristle); the scales on the intervals do not cover the underground completely; striae on the disc broad, but not broader than the intervals, reaching the width of the intervals at the sides of elytra (but often covered by scales); punctures deep and rounded.

Legs. Long; the marginal front femora reach the base of the rostrum, and the hind femora reach the end of the elytral apex. They are covered with predominantly brown scales; tibia with short, white, and laterally protruding bristles.

Venter. The 2nd strip-type sternite only a little bit longer than the 3rd, but not longer than sternites 3 and 4 together. 1st sternite of male with a broad depression (Figure 6(b)).

Female Genital. See Figure 6(n).

Aedeagus. Median lobe of aedeagus small, 1.64x as long as wide; see Figure 6(c).

Etymology. The species name refers to a "Vila" (fairy) in the Velebit Mts. This massif has a similar relevance for Croatians to Olympus for Greeks or the Fuijiyama for the Japanese. In Croatia, the mystical Velebit Mts. range is famous for its fairies, the most celebrated called "Vila Velebita" (The Fairy of Velebit).

Ecology. Onyxacalles vilae was sifted by the first author in the Velebit mountains of Croatia under Fagus and Quercus between 800 and $1600 \mathrm{~m}$ above sea level.

Distribution. A complete distribution map will be given in a separate faunistic study in the future, but this species was also sifted by the author in the Montagne de Lans near Lans en Vercors (France: Isère)—on limestone and under Fagus, too $\left(\mathrm{N} 45^{\circ} 06^{\prime} 45^{\prime \prime} \mathrm{E} 05^{\circ} 36^{\prime} 21^{\prime \prime}, 1352 \mathrm{~m}\right)$. Working hypothesis: It could be possible that all specimens of the "Alpine Arc"between Grenoble and the Velebit mountains-belong to this 
new species and can be separated from the Central Italian populations of Onyxacalles luigionii (cf. Figure 3).

\section{Key to the Species of Onyxacalles Stüben, 1999}

(1) Smaller species with an ovally rounded habitus, legs shorter, femora reach the base of the rostrum; rostrum shorter and broader; if tufts of bristles exist on the elytral slope, these are only densely placed (not tapered). Distribution: continent of Western Palaearctic.

Subgenus: Onyxacalles s. str.......2

$\left(1^{*}\right)$ Larger species with a more "elliptical" habitus and with long legs, femora reach the insertions of the antennae; rostrum very long and slender; the tapered tufts of bristles on the elytral slope strongly protruding (Figures $8(\mathrm{~b})-8(\mathrm{~d})$ ). Distribution: Western Canary Islands.

Subgenus: Araneacalles Stüben and Astrin, $2010 \ldots 16$

(2) Apex of the aedeagus regularly rounded in lateral view: Figures 6(c) and 6(g) (both species without distinctive tufts of bristles on the uneven elytral intervals, only with densely placed bristles in one or two rows).

\section{Luigionii group ........2}

$\left(2^{*}\right)$ Apex of the aedeagus with a second, separated peak in lateral view (cf. Figures 9(a)-9(1)) (most species with more or less characteristic tufts of bristles on the elytra).

\section{— Luigionii group -}

(3) Rostrum of the male more slender, $3.1 \mathrm{x}$ as long as wide; punctures mainly dense towards the apex (only separated by small ridges) (Figure 6(j)). Bristles of high-contrast elytra on the 1st and 3rd intervals shorter, broader (towards the apex of bristle), and more dense (Figures 6(d) and 6(h)). Median lobus of aedeagus broader, $1.93 \mathrm{x}$ as long as wide; apex ("hook") of the aedeagus longer (in ventral view), strongly (nearly rectangular) curved in lateral view. Internal structure (endophallus) of the sac different (Figure 6(g)). Distribution: Central and Southern Italy (Figure 3).

Onyxacalles luigionii (A. \& F. Solari, 1907) [32] $=$ ? Onyxacalles porcheti [39] (Figure 8(e), Pyrenees)

(3*) Rostrum of the male broader, $2.8 \mathrm{x}$ as long as wide (as measured by apex); punctures not so densely packed in front of the apex (separated by flat intervals) (Figure 6(i)). Bristles of the low-contrast elytra on the 1st and 3rd intervals (excluding the sutural stripe) longer and more slender; their distance larger
(Figure 6(a)). Median lobus of aedeagus smaller, $1.64 \mathrm{x}$ as long as wide; apex ("hook") a little bit shorter (in ventral view), flatter, not so strongly curved in lateral view (Figure 6(c)). Distribution: Croatia (l.t.), France: Isère (Figure 3).

Onyxacalles vilae Stüben sp. $\mathbf{n}$.

(4) Pronotum levelled, "triangular" and stubby, strongly broadened just behind the base; the base $2 \mathrm{x}$ longer than the fore-margin (this characteristic is not so pronounced in O. gibraltarensis; however, this species can be clearly separated from all other Onyxacalles s. str. by the completely rounded sides of the aedeagus (Figure 9(a)), see digit 5). Distribution: Southern France, Iberian Peninsula, Morocco.

Portusveneris group 5

$\left(4^{*}\right)$ Pronotum more arched and marginally broader than long, widest at the end of the first third; the base at most $1.5 x$ longer than the fore-margin. Distribution: Algeria, Tunisia, Western Spain, West Mediterranean Islands, Southeastern Europe, Turkey.

\section{- Portusveneris group -}

(5) Pronotum more slender, clearly separated from elytra; body outline broadly similar to the species of the henoni group (see digit $4^{*}$ ), but easy to distinguish from these species by the completely rounded sides of the aedeagus. Habitus (Figure 7(a)). Aedeagus (Figure 9(a)). Distribution: Southern Spain, Northern Morocco (Figure 4).

Onyxacalles gibraltarensis Stüben, 2002

$\left(5^{*}\right)$ Pronotum widest directly behind the base and elytra widest directly in front of the base; therefore, pronotum and elytra do not seem separated (Figures 7(b)$7(\mathrm{e}))$.

........6 6

(6) Elytra egg-shaped towards the apex (Figure 7(b)); Aedeagus (Figure 9(b)). Distribution: Southern France, Iberian Peninsula, Morocco (Figure 4).

Onyxacalles portusveneris (Mayet, 1903) [27]

(6*) Elytra oval or with more or less parallel sides (Figures 7(c)-7(e)).

$\ldots \ldots 7$

(7) Male with a split midtibia spine at the apex; uneven elytral intervals without tufts of bristles. Habitus (Figure 7(c)). Aedeagus (Figure 9(c)). Distribution: Southern Spain (Figure 4).

Onyxacalles seguraensis Stüben, 2003

$\left(7^{*}\right)$ Male without a split midtibia spine at the apex; uneven elytral intervals with tufts of bristles. 


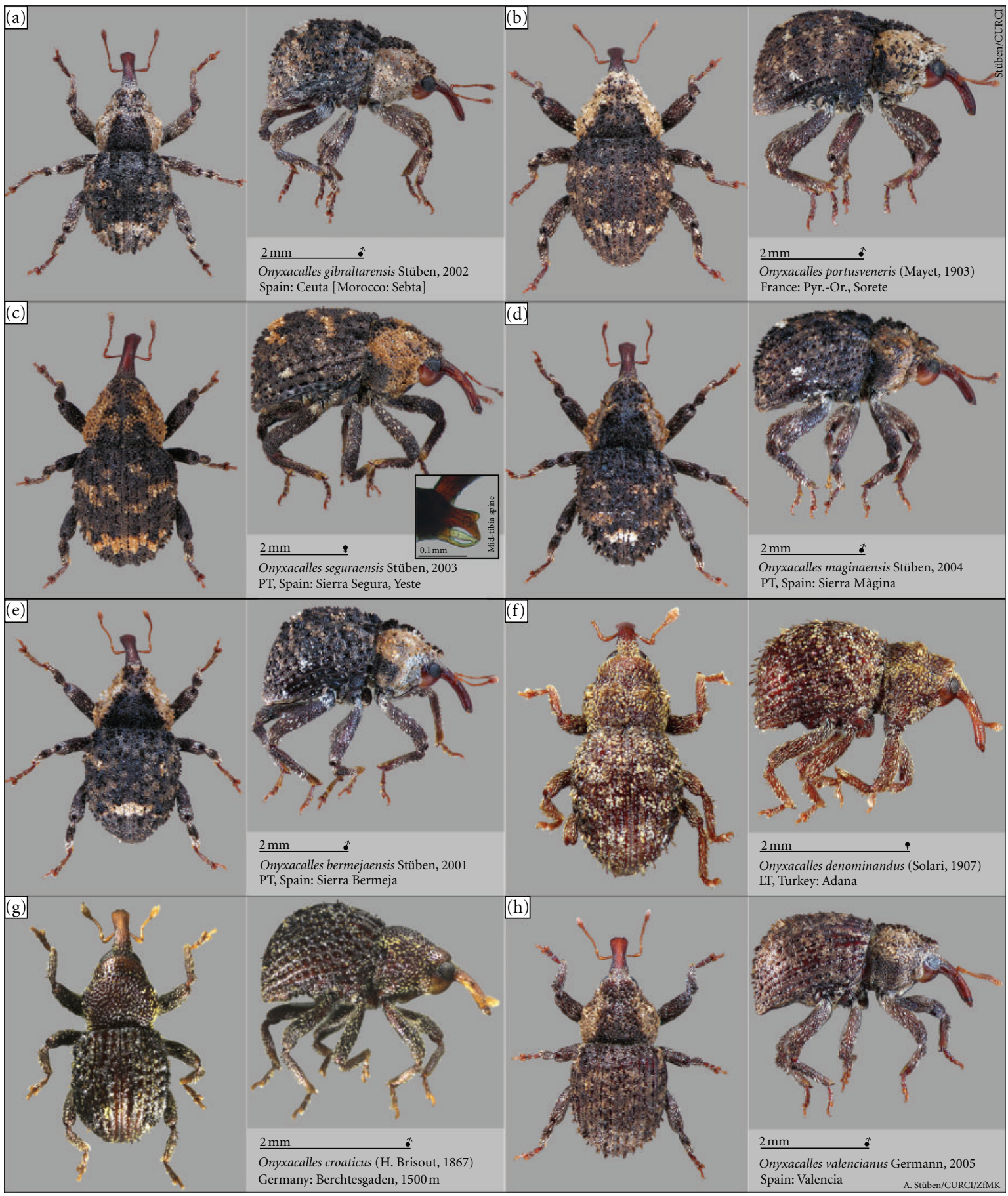

Figure 7: (a-e): Portusveneris group-O.gibraltarensis (a), O. portusveneris (b), O. seguraensis (c), O. maginaensis (d), and O. bermejaensis (e); (f): Incertae sedis-O. denominandus; (g-h): Henoni group (see also next figures)—O. croaticus (g) and O. valencianus (h); all habitus (dor./lat.).

(8) Punctures at the sides of elytra fine and slender; bristles on the first four intervals of the elytral slope in single row. Habitus (Figure 7(d)). Aedeagus (Figure 6(d)). Distribution: Southern Spain (Figure 4).

\section{Onyxacalles maginaensis Stüben, 2004}

$\left(8^{*}\right)$ Punctures at the sides of elytra broader and deeper; bristles on the third interval of the elytral slope densely placed, forming a pronounced tuft at the level of the white fascia. Habitus (Figure 7(e)). Aedeagus (Figure 9(e)). Distribution: Southern Spain (Figure 4).

Onyxacalles bermejaensis Stüben, 2001

(9) Pronotum with a deep midgroove and with strong concavities on each side; a species from Turkey. 


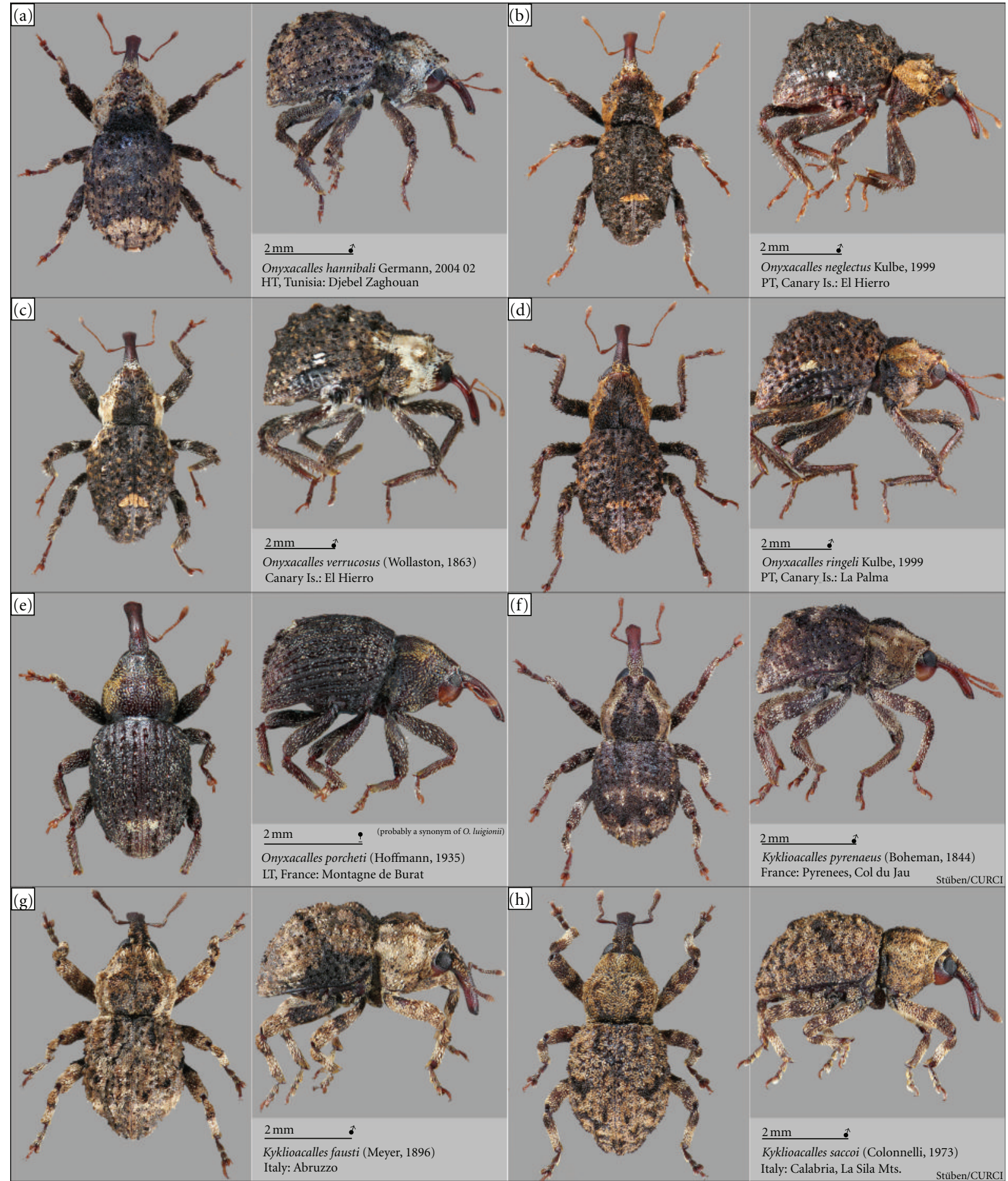

Figure 8: (a): Henoni group-O. hannibali (see also the other species of this group: Figures 5(a), 5(d), 5(g), 5(k)); (b-d): subgenus: Araneacalles -O. neglectus (b), O. verrucosus (c), and O. ringeli (d); (e): O. porcheti (perhaps O. luigionii); (f): Kyklioacalles pyrenaeus; (g) K. fausti; (h) K. saccoi; all habitus (dor./lat.).

Habitus (Figure 7(f)). Aedeagus (Figure 9(f)). Distribution: Turkey.

Onyxacalles denominandus (A. \& F. Solari, 1907) [32] $=$ ? Onyxacalles amasyaensis Wolf, 2001
(9) Pronotum behind the base at most with a flat depression or a hinted channel; mainly West Mediterranean species, only one species from southeastern Europe.

Henoni group 10 


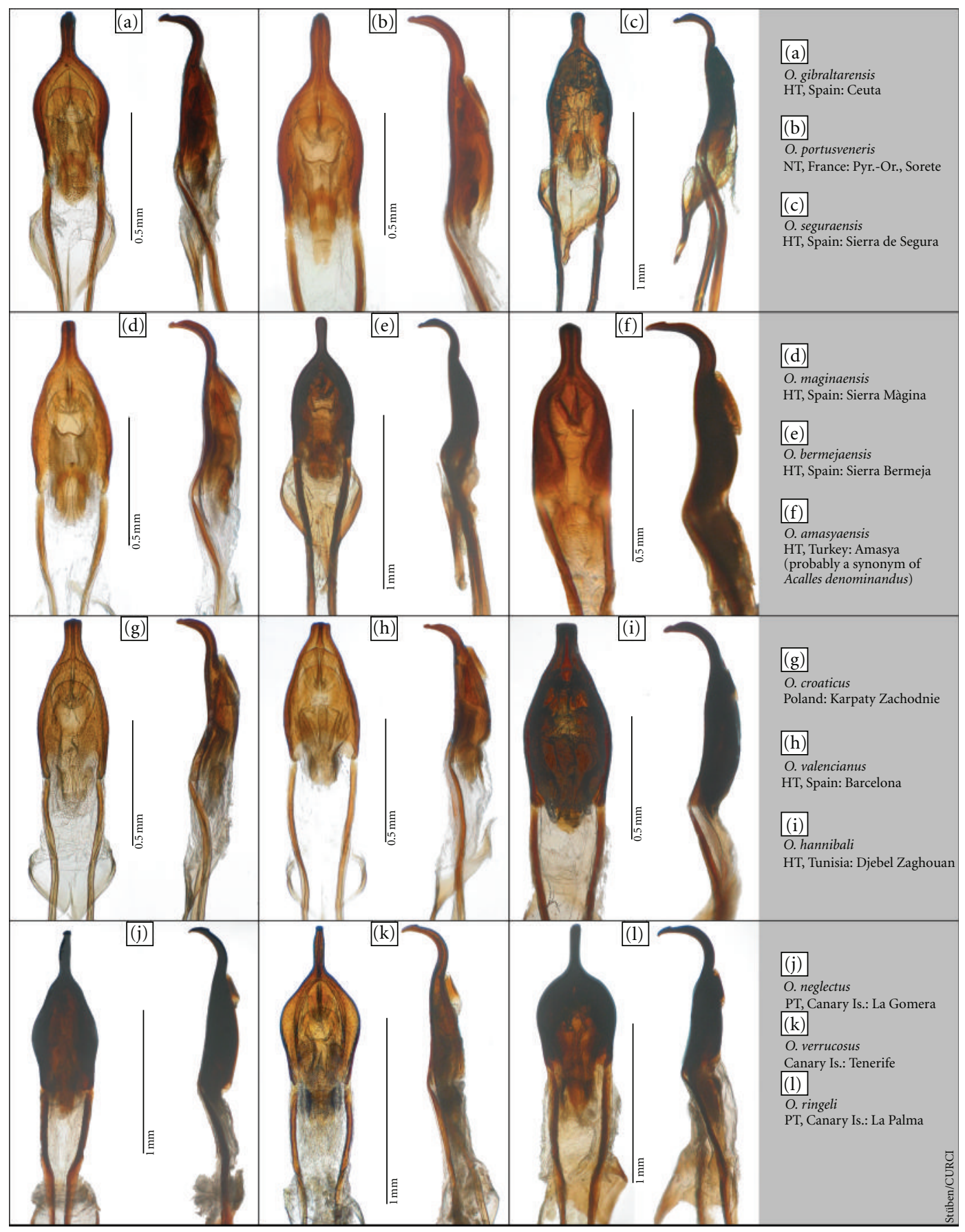

Figure 9: (a-d): Portusveneris group-O. gibraltarensis (a), O. portusveneris (b), O. seguraensis (c), O. maginaensis (d), and O. bermejaensis (e); (f): Incertae sedis-O. amasyaensis; (g-i): Henoni group-O. croaticus (g), O. valencianus (h), O. hannibali (i) (see also the other species of this group: Figures 5(c), 5(f), 5(j), 5(n)); (j-l): subgenus: Araneacalles-O. neglectus (j), O. verrucosus (k), and O. ringeli (l).

\section{- Henoni group -}

(10) Elytra with superelevated and in tubercles dissected intervals; a species from southeastern Europe, which is added to the henoni group preliminary on the basis of a similar form of the aedeagus. Habitus (Figure $7(\mathrm{~g})$ ). Aedeagus (Figure 9(g)). Distribution: East and Southeast Europe [31].
(10) Elytra flat, without tubercles.

(11) Elytra and pronotum (almost) without tufts of bristles; these single, beaded bristles placed in a row. 
$\left(11^{*}\right)$ Elytra on the intervals 1 and 3 and pronotum at the sides with tufts of bristles; these bristles densely placed in 2-3 rows, forming tufts at regular intervals. ........ 13

(12) Elytral bristles short, shovel-shaped and densely placed (at most $1.3 \mathrm{x}$ as long as wide); elytra rich in contrast (colored); apex of aedeagus strongly curved in lateral view. Habitus (Figure 5(g)). Aedeagus (Figure 5(j)). Distribution: Spain, Majorca (Figure 2).

\section{Onyxacalles balearicus Stüben, 2005}

$\left(12^{*}\right)$ Elytral bristles more slender, at least $2 \mathrm{x}$ as long as wide and their distance large; elytra poor in contrast; apex of aedeagus flatter curved in lateral view. Habitus (Figure 6(n)). Aedeagus (Figure 9(h)). Distribution: Eastern Spain (Figure 2).

\section{Onyxacalles valencianus Germann, 2005}

(13) Disc of pronotum with a channel from the base towards the flat sector in front of the fore-margin; elytra of male with parallel sides in the middle sector (dorsal view). Habitus (Figure 5(a)). Aedeagus (Figure 5(c)). Distribution: Italy, Sardinia (Figure 2).

\section{Onyxacalles nuraghi Stüben sp. n.}

(13) Disc of pronotum at most with a flat hallow behind the base; elytra of male broader and stronger (short ovally) rounded (slightly "egg-shaped").

....... 14

(14) Elytral intervals only with a few bristles and small tufts; elytra poor in contrast. Habitus (Figure 8(a)). Aedeagus (Figure 9(i)). Distribution: Tunisia (Figure 2).

Onyxacalles hannibali Germann, 2004

(14*) Elytral intervals studded with bristles and with numerous distinctive tufts; elytra rich in contrast.

15

(15) Rostrum coarsely and densely punctuated towards apex (Figure 5(1)); scales of the elytra predominantly bright: white, beige, or brown (Figure 5(k)); Apex ("hook") of the aedeagus smaller in ventral view and not so strongly curved in lateral view (Figure 5(n)). Distribution: Algeria, Mt. Edough (Figure 2).

\section{Onyxacalles henoni [38]}

$\left(15^{*}\right)$ Rostrum finely punctuated towards apex (Figure $5(\mathrm{~m})$ ); scales of the elytra predominantly dark brown or black (Figure 5(d)); apex ("hook") of the aedeagus broader in ventral view and strongly curved (nearly rectangular) in lateral view (Figure 5(f)). Distribution: France, Corsica (Figure 2).

Onyxacalles torre Stüben and Astrin sp. $\mathbf{n}$.

\section{— Subgenus: Araneacalles -}

(16) Punctures of the 1st and 2nd elytral striae rounded, pothole-like, and as wide as the intervals; the underground of elytra in front of the middle on the 8th and 9th intervals with scales, not shiny; foremargin of pronotum with a curved up collar. Habitus (Figure 8(b)). Aedeagus (Figure 9(j)). Distribution: Canary Is., La Gomera (1.t.), El Hierro (Figure 4).

Onyxacalles neglectus Kulbe, 1999

$\left(16^{*}\right)$ Punctures of the 1st and 2nd elytral striae elongated, clearly smaller than intervals; the underground of elytra in front of the middle on the 8th and 9th intervals without scales, shiny; fore-margin of pronotum without a curved up collar.

........ 17

(17) Punctures at the extreme striae slender, the intervals broader; elytra widest in front of the middle, egg-shaped. Habitus (Figure 8(c)). Aedeagus (Figure 9(k)). Distribution: Canary Is., Tenerife (l.t.), El Hierro (Figure 4).

Onyxacalles verrucosus (Wollaston, 1863) [40]

$\left(17^{*}\right)$ Punctures at the extreme striae larger and rounded, not broader than intervals; elytra oval, widest in the middle. Habitus (Figure 8(d)). Aedeagus (60). Distribution: Canary Is., La Palma (Figure 4).

Onyxacalles ringeli Kulbe, 1999

\section{Acknowledgments}

This paper was prepared by the Molecular Weevil Identification project (MWI) of the CURCULIO Institute (CURCI) and Zoologisches Forschungsmuseum Alexander Koenig (ZFMK). Keith Bensusan (Gibraltar) kindly revised the English text, and Christina Blume performed the lab work.

\section{References}

[1] P. E. Stüben, "Die westpaläarktischen Arten der Gattung Onyxacalles g.n. (Col., Curculionidae: Cryptorhynchinae)," Entomologische Blätter, vol. 95, pp. 175-203, 1999.

[2] P. E. Stüben, "Die Arten des Genus Onyxacalles von den Kanarischen Inseln (Curculionidae: Cryptorhynchinae)," Snudebiller, vol. 1, pp. 106-113, 2000.

[3] P. E. Stüben and J. J. Astrin, "Molecular phylogeny in endemic weevils: revision of the genera of Macaronesian Cryptorhynchinae (Coleoptera: Curculionidae)," Zoological Journal of the Linnean Society, vol. 160, no. 1, pp. 40-87, 2010.

[4] P. E. Stüben and I. Wolf, "Beschreibung neuer westpaläarktischer Onyxacalles-Arten. Mit einem neuen Katalog und Schlüssel der Arten des Genus Onyxacalles. (Col.: Curculionidae: Cryptorhynchinae)," Snudebiller, vol. 2, pp. 143$154,2001$.

[5] P. E. Stüben, "Beschreibung neuer Cryptorhynchinae aus Marokko (Coleoptera: Curculionidae)," Snudebiller, vol. 3, pp. 196-225, 2002. 
[6] P. E. Stüben, "Beschreibung neuer Cryptorhynchinae aus Spanien, Italien und Marokko-mit einem neuen Onyxacalles-und Echinodera-Schlüssel (Coleoptera: Curculionidae)," Snudebiller, vol. 4, pp. 186-224, 2003.

[7] Ch. Germann and P. E. Stüben, "Beschreibung neuer Onyxacalles-Arten aus dem mediterranen Raum und Anmerkungen zur Synonymie einiger Arten der Gattung Onyxacalles Stüben 1999. (Coleoptera: Curculionidae: Cryptorhynchinae)," Snudebiller, vol. 5, pp. 116-131, 2004.

[8] P. E. Stüben, "Die Cryptorhynchinae-Fauna der Baleareninsel Mallorca (Coleoptera: Curculionidae)," Snudebiller, vol. 6, pp. 114-130, 2005.

[9] P. E. Stüben, F. Bahr, Ch. Germann, L. Behne, and Ch. Bayer, "Beschreibung neuer Cryptorhynchinae aus dem mediterranen Raum (Coleoptera: Curculionidae)," Snudebiller, vol. 6, pp. 84-113, 2005.

[10] H. Brisout de Barneville, "Nouveaux tableau des Acalles avec la description de deux nouvelles espèces et celle de l'Orchestes quedenfeltii Gerhard," Annales de la Société Entomologique de France, vol. 4, no. 7, pp. 57-64, 1867.

[11] P. E. Stüben and V. Thurn, "Die Wälder sind der Menschheit vorausgegangen, die Wüsten folgen ihr," in WüstenErde. Der Kampf Gegen Durst, Dürre und Desertifikation, P. E. Stüben and V. Thurn, Eds., pp. 16-28, Ökozid 7, Gießen, Germany, 1991.

[12] V. ThurnP. E. Stüben and V. Thurn, "Die Wüste greift nach Europa. Die Zerstörung der Mittelmeerwälder," in WüstenErde. Der Kampf gegen Durst, Dürre und Desertifikation, pp. 121-136, Ökozid 7, Gießen, Germany, 1991.

[13] P. E. Stüben, K. Fabian, and J. J. Astrin, TreeClimbers. Den-droacalles, Silvacalles and Lauriacalles of the Canary Islands (Curculionidae: Cryptorhynchinae), CURCULIO-Institute, Mönchengladbach, Germany, 2009.

[14] P. E. Stüben, L. Behne, A. Floren et al., Canopy Fogging in the Canarian laurel forest of Tenerife and La Gomera, CURCULIOInstitute, Mönchengladbach, Germany, 2010.

[15] B. Dayrat, "Towards integrative taxonomy," Biological Journal of the Linnean Society, vol. 85, no. 3, pp. 407-415, 2005.

[16] K. W. Will, B. D. Mishler, and Q. D. Wheeler, "The perils of DNA barcoding and the need for integrative taxonomy," Systematic Biology, vol. 54, no. 5, pp. 844-851, 2005.

[17] A. D. Roe and F. A. H. Sperling, "Population structure and species boundary delimitation of cryptic Dioryctria moths: an integrative approach," Molecular Ecology, vol. 16, no. 17, pp. 3617-3633, 2007.

[18] J. M. Padial, S. Castroviejo-Fisher, J. Köhler, C. Vilà, J. C. Chaparro, and I. D. I Riva, "Deciphering the products of evolution at the species level: the need for an integrative taxonomy," Zoologica Scripta, vol. 38, no. 4, pp. 431-447, 2009.

[19] M. Heethoff, M. Laumann, G. Weigmann, and G. Raspotnig, "Integrative taxonomy: combining morphological, molecular and chemical data for species delineation in the parthenogenetic Trhypochthonius tectorum complex (Acari, Oribatida, Trhypochthoniidae)," Frontiers in Zoology, vol. 8, article 2, 2011.

[20] D. S. H. Tan, Y. Ang, G. S. Lim, M. R. B. Ismail, and R. Meier, "From "cryptic species" to integrative taxonomy: an iterative process involving DNA sequences, morphology, and behaviour leads to the resurrection of Sepsis pyrrhosoma (Sepsidae: Diptera)," Zoologica Scripta, vol. 39, no. 1, pp. 5161, 2010.

[21] J. J. Astrin, P. E. Stüben, B. Misof et al., "Exploring diversity in cryptorhynchine weevils (Coleoptera) using distance-, character- and tree-based species delineation," Molecular Phylogenetics and Evolution, vol. 63, no. 1, pp. 1-14, 2012.

[22] Th. S. Kuhn, The Structure of Scientific Revolutions, University of Chicago Press, Chicago, Ill, USA, 1st edition, 1962.

[23] O. Folmer, M. Black, W. Hoeh, R. Lutz, and R. Vrijenhoek, "DNA primers for amplification of mitochondrial cytochrome c oxidase subunit I from diverse metazoan invertebrates," Molecular Marine Biology and Biotechnology, vol. 3, no. 5, pp. 294-299, 1994.

[24] J. J. Astrin and P. E. Stüben, "Phylogeny in cryptic weevils: molecules, morphology and new genera of Western Palaearctic Cryptorhynchinae (Coleoptera: Curculionidae)," Invertebrate Systematics, vol. 22, no. 5, pp. 503-522, 2008.

[25] P. Meyer, "Diagnose sechs neuer Acalles-Arten der palaearctischen Region, nebst einigen synonymischen Bemerkungen über die Gattung. (Gegeben als Vorarbeit zu einer Bestimmungs-Tabelle der Cryptorrhynchiden Europa's und der angrenzenden Länder)," Wiener entomologische Zeitung, vol. 15, pp. 13-16, 1896.

[26] C. H. Boheman and J. C. Schoenherr, "new taxa," in Genera et species curculionidum, cum synonymia hujus familiae, vol. 7 of pars I. Supplementum, Roret, Fleischer, Paris, Farnce, 1844.

[27] V. Mayet, "Contribution a la faune entomologique des pyrénées orientales," Coléoptères des Albères. Miscelánea Zoológica, vol. 11, pp. 67-76, 1903.

[28] R. C. Edgar, "MUSCLE: a multiple sequence alignment method with reduced time and space complexity," BMC Bioinformatics, vol. 5, article 113, 2004.

[29] C. Lanave, G. Preparata, C. Saccone, and G. Serio, "A new method for calculating evolutionary substitution rates," Journal of Molecular Evolution, vol. 20, no. 1, pp. 86-93, 1984.

[30] F. Ronquist and J. P. Huelsenbeck, "MrBayes 3: bayesian phylogenetic inference under mixed models," Bioinformatics, vol. 19, no. 12, pp. 1572-1574, 2003.

[31] P. E. Stüben, "An illustrated up-to-date catalogue of Westpalearctic Cryptorhynchinae (Curculionoidea)," 2012, http:// www.curci.de/illustrated_catalogue/westpalearctic_cryptorhynchinae/.

[32] A. Solari and F. Solari, "Studi sugli Acalles," Annali del Museo Civico di Storia Naturale "G. Doria” (3), vol. 3, no. 43, pp. 479551, 1907.

[33] M. Košvtál and M. Holecová, "Neotype designation of Onyxacalles pyrenaeus and description of its sibling species Onyxacalles boehmei sp. nov. from Central Europe (Coleoptera: Curculionidae)," Entomological Problems, vol. 32, no. 1, pp. 73-78, 2001.

[34] P. E. Stüben and J. J. Astrin, "Molecular phylogeny of the weevil genus Kyklioacalles Stüben, with descriptions of a new subgenus Glaberacalles and two new species (Curculionidae: Cryptorhynchinae)," Zootaxa, no. 2662, pp. 28-52, 2010.

[35] E. Colonnelli, "Una nuova specie di Acalles Schönherr del Massiccio del Pollino (Coleoptera, Curculionidae)," Bollettino dell 'Assoziazione Romana di Entomologia, vol. 27, no. 1-2, pp. 25-30, 1973.

[36] P. E. Stüben, “Taxonomie und Phylogenie der westpaläarktischen Arten der Gattung Kyklioacalles g.n. (Coleoptera, Curculionidae: Cryptorhynchinae)," Stuttgarter Beiträge zur Naturkunde Serie A, vol. 584, p. 38, 1999.

[37] J. W. Wägele, Foundations of Phylogenetic Systematics, Pfeil, Munich, Germany, 2005.

[38] L. Bedel, "Description d'une Curculionide nouveau d'Algérie," Bulletin de la Société Entomologique de France, vol. 8, article xxxvi, 1888. 
[39] A. Hoffmann, "Description d'un Acalles nouveau de France (Col., Curculionidae)," Bulletin de la Société Entomologique de France, vol. 40, pp. 162-164, 1935.

[40] T. V. Wollaston, "Diagnostic notices of new Canarian Coleoptera," Annals Magazine of Natural History (3), vol. 11, pp. 214-221, 1863. 

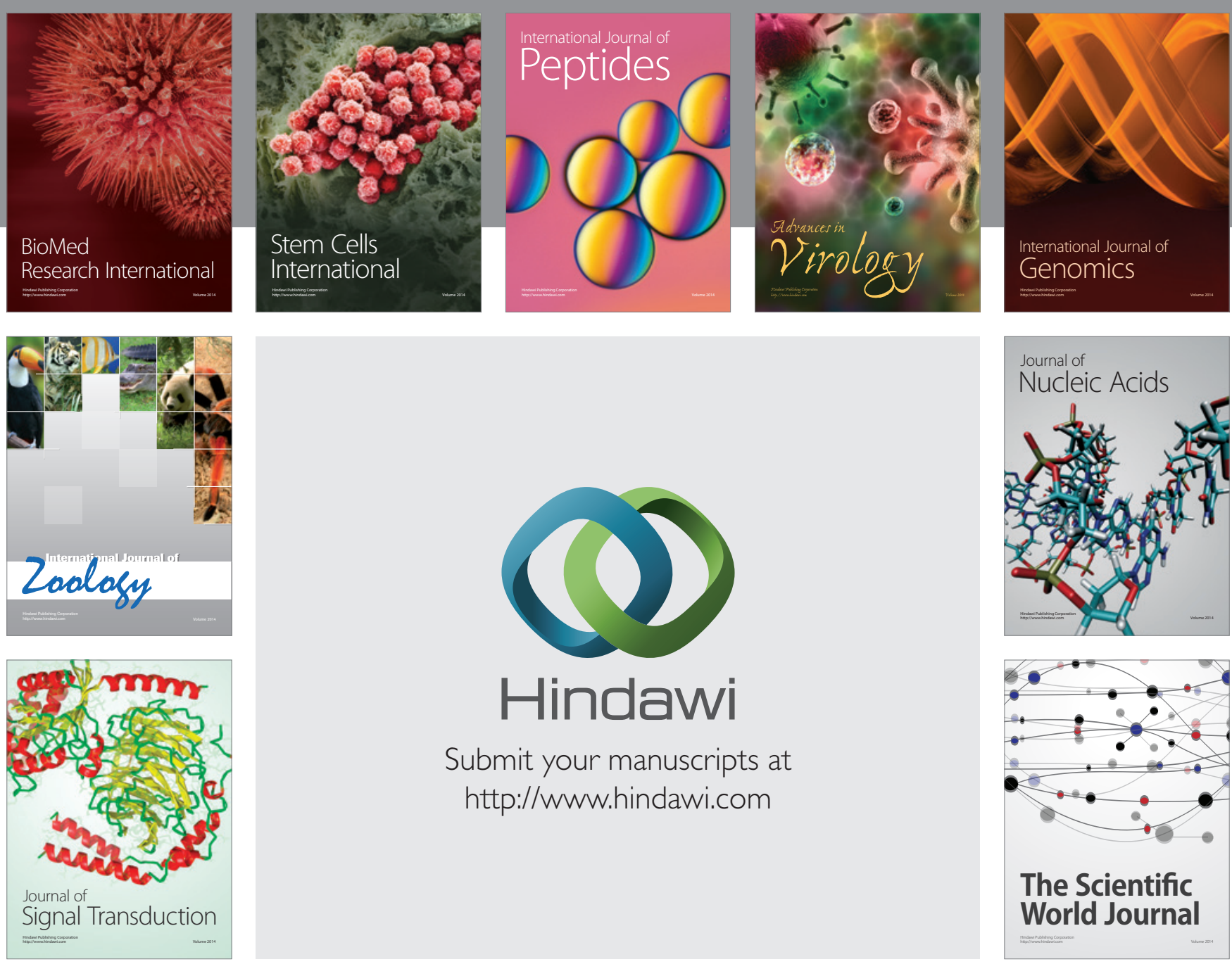

Submit your manuscripts at

http://www.hindawi.com
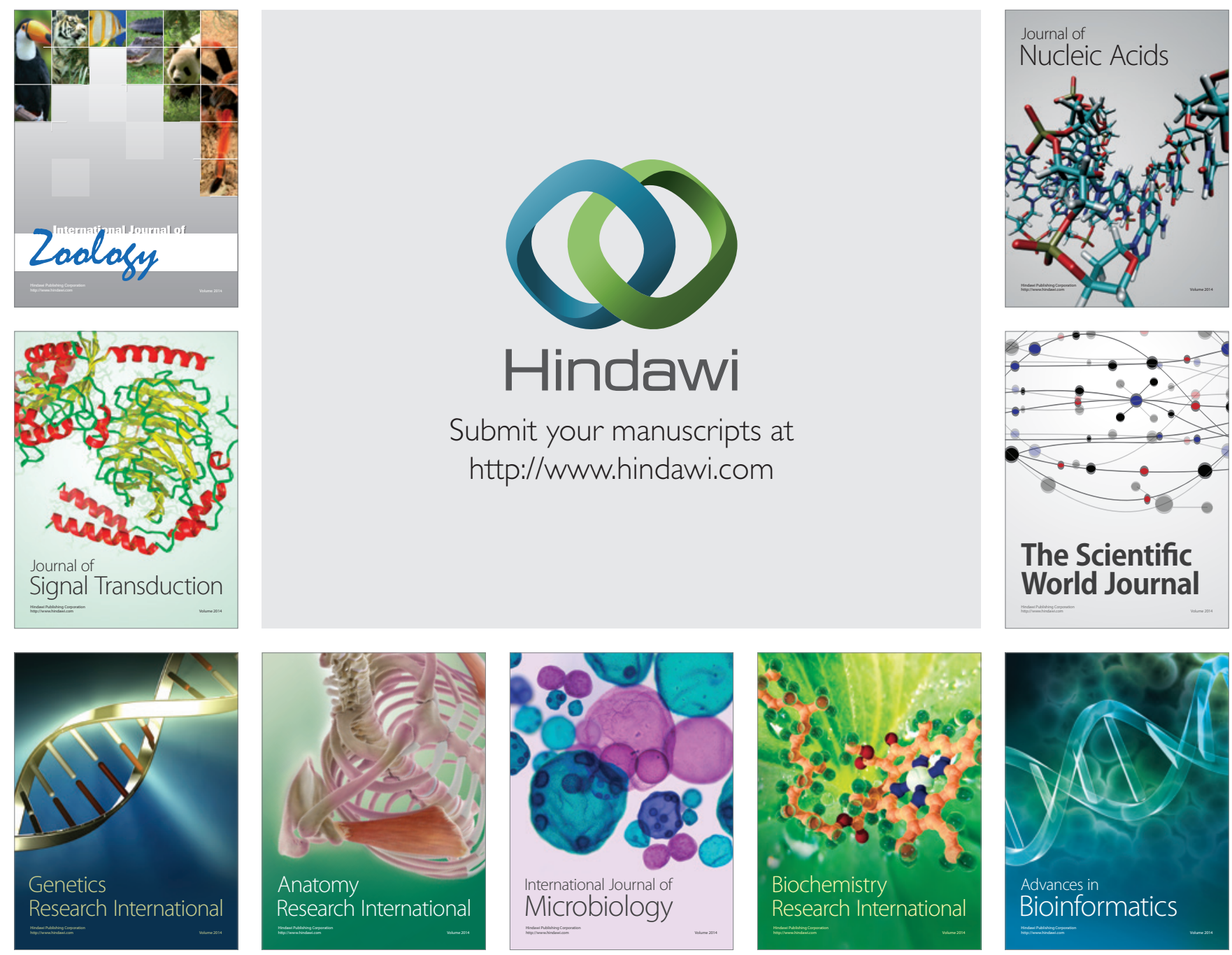

The Scientific World Journal
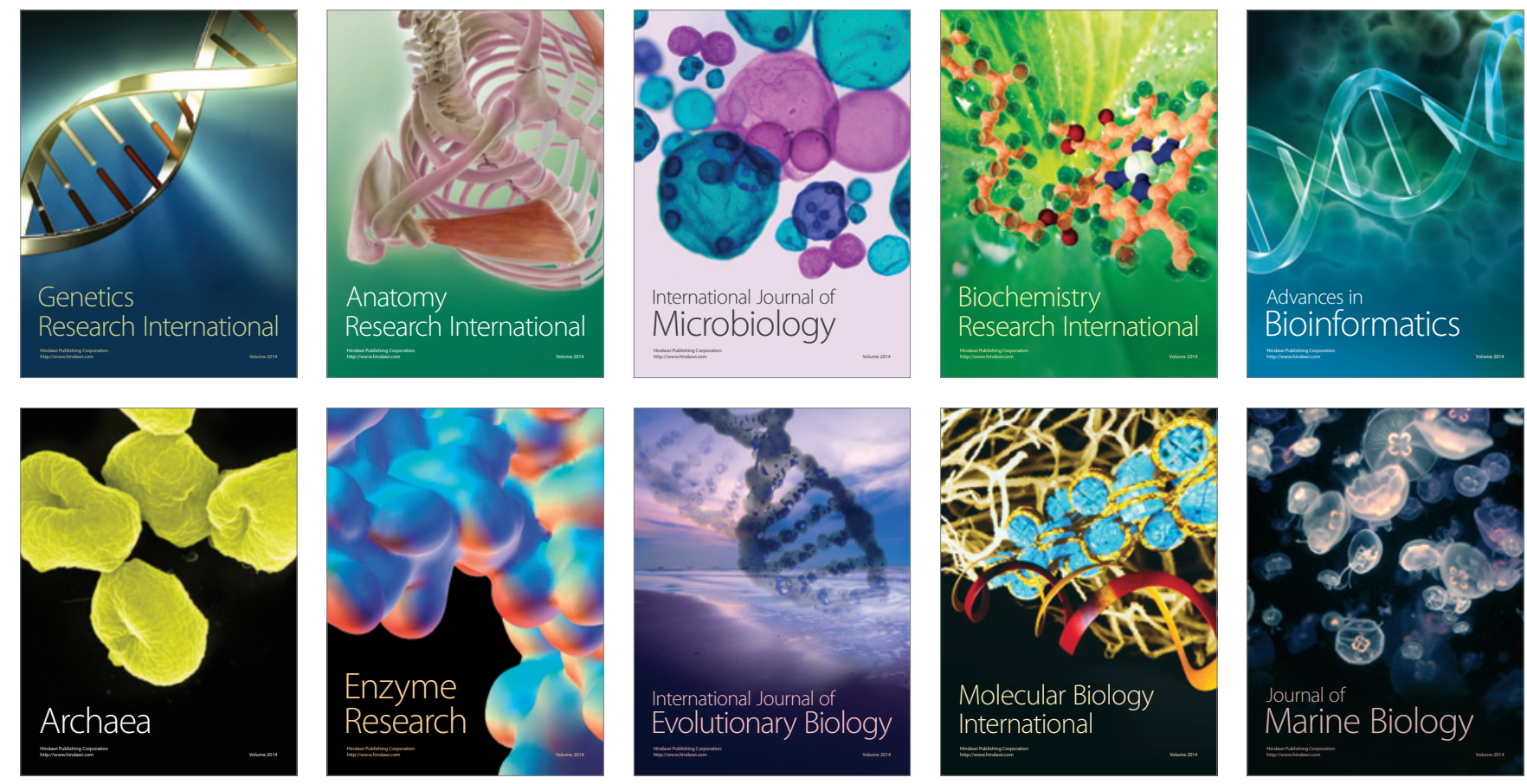